\title{
Benefits of Improper Gaussian Signaling in Interweave Cognitive Radio with Full and Partial CSI
}

\author{
Wafa Hedhly, Student Member, IEEE, Osama Amin, Senior Member, IEEE, and Mohamed-Slim Alouini, \\ Fellow, IEEE
}

\begin{abstract}
In this paper, we investigate the impact of using improper Gaussian signaling (IGS) scheme on the interweave cognitive radio $(\mathrm{CR})$ paradigm when the cognitive user has access to full or partial channel-state-information (CSI). Throughout this work, we analyze the performance of both the primary user (PU) and secondary user (SU) in terms of instantaneous achievable rate and outage probability, for given sensing and detection capabilities. Then, we optimize the IGS scheme for full and partial CSI scenarios to maximize the achievable rate of the SU while maintaining a minimum quality-of-service $(\mathrm{QoS})$ requirement for the $\mathrm{PU}$. Meanwhile, we derive the corresponding feasibility conditions for the interweave cognitive radio system operation. IGS based systems are proven to provide notable gain over the conventional proper Gaussian signaling, which is considered our benchmark scheme. The IGS scheme is observed to be further beneficial at low SU detection capabilities, strong interference from the cognitive user to the licensed user, and low gain of the PU direct link.
\end{abstract}

Index Terms-Interweave, cognitive radio, improper Gaussian signaling, asymmetric signaling, miss detection, interference channels.

\section{INTRODUCTION}

In the last few years, the evolution of wireless communications caused a shortage in spectral resources, the latter becoming scarce and expensive. The spectrum is not really overpopulated but indeed inefficiently exploited. In fact, according to the fixed spectrum access policy currently used in spectrum regulation, each bandwidth is assigned exclusively to licensed users regardless of the actual communication activity [1]. Therefore, the saturation of the spectrum can be avoided through effective spectrum management policies. To this end, cognitive radio (CR) can be seen as a promising alternative of spectrum access approaches that can minimize wasting spectrum resources. The three different paradigms for $\mathrm{CR}$ are known as underlay, overlay and interweave through which accessing the spectrum of licensed users and taking advantage of the unused holes become possible [2]. The underlay paradigm allows secondary users (SUs) to access the spectrum if their transmitted power does not cause destructive interference to primary transmission. In the overlay cognitive radio, the SU exploits his prior knowledge of the primary user (PU) message or codebook to cancel its interference or even to assist the PU communication. The interweave CR is an

The authors are with CEMSE Division, King Abdullah University of Science and Technology (KAUST), Thuwal, Makkah Province, Saudi Arabia. E-mail: $\{$ wafa.hedhly, osama.amin, slim.alouini\}@kaust.edu.sa opportunistic spectrum access paradigm where the SU detects the presence or the absence of the PU, to identify spectrum holes (space, time or frequency voids) and communicate with reduced interference to the primary user [2]. In this regard, the interweave CR needs accurate sensing and detection systems to avoid any interference to the authorized users.

The interweave CR overall system performance is significantly affected by the miss-detection and false-alarm events. Thus, studying the interweave CR system requires taking into account these two limiting parameters. Furthermore, developing different detection systems can affect system performance such as energy detection [3], [4], [5] and matched filter [6], [7]. Different research work have been conducted to implement interweave CR in interference-limited networks [8], [9]. In [8], Kaushik et al. improved the achievable rate of the SU by tuning the sensing and estimation parameters while keeping a certain probability of detection. In [9], the detection procedure is optimized to improve the SU communication in both underlay and interweave CR systems to keep a certain PU outage probability. Moreover, the detection procedure can take a very long time if the PU direct link is weak [2]. Therefore, under some circumstances, the efficiency of the detection system is not guaranteed, leading to severe deterioration of the licensed user's performance. Throughout this paper, we adopt the so called improper Gaussian signaling (IGS) as a CR signaling scheme to improve its performance.

IGS or asymmetric signal can have correlated real and imaginary Gaussian variables, with possibly unbalance in the power distribution between them [10], [11], which is a relaxation of the proper Gaussian signal (PGS) assumption. A comprehensive survey was conducted recently and discussed the basic background for improper Gaussian signals, performance analysis, system design and practical realization in [12]. As explained in [13], we can obtain improper signals from proper signals via widely linear transformation. IGS has been envisaged as a promising signaling scheme in interferencelimited networks, to reliably reduce the impact of interference signals [14]-[20], which can be seen as interference alignment approach via arranging signals space [21]. As a result, the impropriety degree has been introduced as an additional design parameter that can tackle the impact of interference and improve system performance. Improper signals are found to provide significant improvement in the performance of several systems [14]-[16], [19]. Maximizing the achievable rate of interference-limited networks was a widely investigated 
research topic, exploiting the benefits of improper signals in several scenarios such as Single-Input-Single-Output (SISO) communication [14]-[16], [19], Non-Orthogonal Multiple Access (NOMA) systems [22], full-Duplex relay systems [10], [23], systems undergoing hardware impairments [24]-[28], etc. The benefits of IGS in interference-limited networks grant more insights to employ improper signaling in CR networks.

In cognitive radio networks, the presence of the SU can deteriorate the performance of the PU communication. However, this main limitation to $\mathrm{CR}$ networks can be mitigated using IGS, that can reduce the interference effect in such systems through the use of appropriate resource allocation schemes [29]-[34]. IGS enhances the operation of cognitive radio systems by making the impact of the SU signal on the PU performance less significant [29], [32], [33]. Interesting works have been conducted to implement underlay and overlay networks using IGS. In [29], Lameiro et al. considered a SISO underlay cognitive radio network composed of primary and secondary users in an interference-limited scenario. Using an improper secondary signal helps to improve the performance of the system significantly, by reducing the interference impact on the PU through achievable rate analysis. Furthermore, in [30], the benefit of employing IGS was highlighted, where statistical distribution for the SU achievable rate was derived given a fixed SNR at the PU side. A full-duplex communication was considered in [32] with an additional self-interference at the PU transmitter and receiver, reducing the opportunities of the SU communication and giving more insights to use IGS. An outage probability analysis has been performed to reduce the outage probability of the SU while meeting a minimum quality of service for the PU. Additionally, in [33], Amin et al. explored the benefits of employing IGS in overlay cognitive radio systems first, when the secondary transmitter has a full channel state information (CSI) and then with partial channel knowledge. The results showed that the IGS outperforms the PGS mainly with a strong interference from the SU to the $\mathrm{PU}$, in terms of SU achievable rate. Moreover, physical layer security in CR networks is another application of improper signals. Employing an eavesdropper aware IGS scheme for the SU helps to improve the secrecy system performance, which is done by minimizing the secrecy outage probability [35]. According to the recent IGS survey [12], interweave $\mathrm{CR}$ has not received research interest as underlay and overlay CR, which motivated us to study the impact of IGS on the interweave CR system.

In this paper, we study the performance of interweave cognitive radio system when the SU is sending IGS for known miss-detection and false alarm probabilities with full or partial CSI knowledge ${ }^{1}$. The PU sends proper signals while the SU employs IGS to reduce the impact of its interference on the primary message. The limitation brought by the sensing errors in interweave systems can cause harmful interference to the licensed communication. Therefore, at low sensing and detection capabilities, the impropriety degree of the SU signal serves as a second degree of freedom, in addition to the transmit power, to enhance the SU communication while

\footnotetext{
${ }^{1}$ The full CSI analysis and design part was partially presented in [34].
}

maintaining a reliable performance for the PU. To the best of the authors' knowledge, the benefit of employing IGS in interweave $\mathrm{CR}$, in the partial-CSI case and the comparison with the full CSI case, have not been analyzed in the literature. Therefore, it is important to test whether employing IGS in a simple setup of single user, is beneficial, before considering multiuser case. In the following, we summarize the main contribution as,

- Derive the expressions of the achievable rate and the outage probability of both the PU and the SU in interweave CR system when the SU uses IGS while the PU uses PGS.

- Optimize the SU signal parameters to maximize its achievable rate while maintaining acceptable QoS for the PU for a fixed miss-detection and false-alarm probabilities, assuming full or partial CSI.

- Compare through numerical results, the benefits obtained from adopting IGS over PGS, assuming different system parameters in the interweave CR paradigm for both full and partial CSI scenarios.

The rest of this paper is organized as follows: in section II, we present the interweave system model. Then, we study the achievable rate and outage probability performance in section III. In sections IV and V, we analyze and solve the perfect and partial CSI based design problems, respectively. After that, we provide extensive simulation results in section VI, for both perfect and partial CSI based scenarios. Finally, we conclude the paper in section VII.

\section{Interweave Cognitive Radio System Model}

Consider an interweave CR system depicted in Fig. 1, where the spectrum is assigned to a single licensed user PU. A cognitive radio network is composed of single unlicensed user that senses and accesses the spectrum whenever it is free. We assume that both the PU and SU operate in half-duplex mode.

The primary network is composed of a primary transmitter (PT) communicating with a primary receiver (PR) using a transmit power $p_{\mathrm{p}}$. The $\mathrm{SU}$ accesses the spectrum using a transmit power $p_{\mathrm{s}}$. We denote by $h_{\mathrm{XY}}$ the channel coefficient from the transmitter $X$ to the receiver $Y$ where $X, Y \in\{s, p\}$. In particular, $h_{\mathrm{XX}}$ and $h_{\mathrm{XY}}$ are respectively a direct and a cross channel. The overall network is subject to additive white Gaussian noise (AWGN) with zero mean and variance $\sigma^{2}$. We define the channel-to-noise ratios $(\mathrm{CNR})$ as $\gamma_{\mathrm{X}}=\left|h_{\mathrm{XX}}\right|^{2} / \sigma^{2}$ and $\mathcal{I}_{\mathrm{Y}}=\left|h_{\mathrm{XY}}\right|^{2} / \sigma^{2}$ for direct and interference links respectively.

In this interweave system, the coexistence of primary and secondary signals is not allowed and this feature is controlled via the probability of detection $P_{\mathrm{d}}$ and the probability of false alarm $P_{\mathrm{fa}}$. Using the state of operations of the primary and secondary transmitters, denoted respectively $S_{\mathrm{p}}$ and $S_{\mathrm{s}}$, we can define the probability of detection and the probability of false alarm as [36],

- $P\left(S_{\mathrm{s}}=1 / S_{\mathrm{p}}=1\right)=1-P_{\mathrm{d}}$

- $P\left(S_{\mathrm{s}}=0 / S_{\mathrm{p}}=1\right)=P_{\mathrm{d}}$

- $P\left(S_{\mathrm{s}}=1 / S_{\mathrm{p}}=0\right)=1-P_{\mathrm{fa}}$

- $P\left(S_{\mathrm{s}}=0 / S_{\mathrm{p}}=0\right)=P_{\mathrm{fa}}$, 


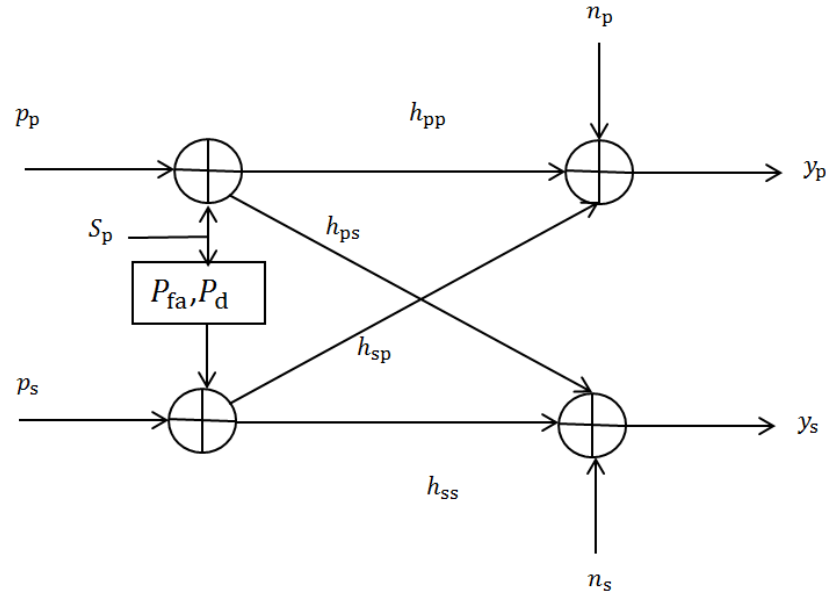

Figure 1: System model

where the inactivity of the PU occurs with $P\left(S_{\mathrm{p}}=0\right)=p$ that reduces the activity probability to be $P\left(S_{\mathrm{p}}=1\right)=1-p$. To characterize the proper Gaussian signaling and the improper Gaussian signaling, we present the following definitions.

Definition 1: [11], [13] If the pseudo-variance of a complex random variable $x$ is zero i.e. $\mathbb{E}\left[x^{2}\right]=0, x$ is called proper, otherwise $x$ is improper.

Definition 2: [13], [29] We define the circularity coefficient of a zero mean scalar random variable $x$ as, $\mathcal{C}_{x}=$ $\left|\mathbb{E}\left[x^{2}\right]\right| / \mathbb{E}\left[|x|^{2}\right]$, where $0 \leq \mathcal{C}_{x} \leq 1$. This coefficient measures the impropriety degree of the random variable. If $\mathcal{C}_{x}=0$, $x$ is proper and $\mathcal{C}_{x}=1$ characterizes a maximally improper variable.

\section{Performance Analysis}

\section{A. Achievable rate Performance}

In this section, we derive expressions for the achievable rate of the PU and the SU, considering the sensing and detection parameters, in interweave cognitive radio system. The PU is assumed to adopt PGS scheme since it is not aware of the presence of the interfering secondary signal when accessing its licensed band. Contrarily, we assume the SU is using IGS scheme and is capable of tuning its signaling parameters. The activity of the primary user and the detection parameters affect the achievable rate of the PU and the SU. The operation of the cognitive user is defined considering two events: the presence of the primary signal with a probability of $(1-p)\left(1-P_{\mathrm{d}}\right)$ and its absence with a probability of $p\left(1-P_{\mathrm{fa}}\right)$. Hence, we can express the $\mathrm{SU}$ achievable rate as follows,

$R_{\mathrm{S}}\left(p_{\mathrm{s}}, \mathcal{C}_{x}\right)=\frac{T-\tau}{T}\left(p\left(1-P_{\mathrm{fa}}\right) R_{\mathrm{S}_{1}}+(1-p)\left(1-P_{\mathrm{d}}\right) R_{\mathrm{s}_{2}}\right)$,

where $\tau$ denotes the sensing time and $T$ the allocated time for each packet. As mentioned before, the achievable rate of the
SU depends on two events, giving two expressions $R_{\mathrm{s}_{1}}$ and $R_{\mathrm{S}_{2}}$ in (1), written as,

$$
\begin{aligned}
& R_{\mathrm{s}_{1}}=\log _{2}\left(1+p_{\mathrm{s}} \gamma_{\mathrm{s}}\right)+\frac{1}{2} \log _{2}\left(1-\mathcal{C}_{\mathrm{ss} 1}^{2}\right) \\
& R_{\mathrm{s}_{2}}=\log _{2}\left(1+\frac{p_{\mathrm{s}} \gamma_{\mathrm{s}}}{1+p_{\mathrm{p}} \mathcal{I}_{\mathrm{p}}}\right)+\frac{1}{2} \log _{2}\left(1-\mathcal{C}_{\mathrm{ss} 2}^{2}\right),
\end{aligned}
$$

where $\mathcal{C}_{\mathrm{ss} 1}$ and $\mathcal{C}_{\mathrm{ss} 2}$ denote the circularity coefficients of the $\mathrm{SU}$ received signals for the two events. These two coefficients are expressed, according to Definition 2, as,

$$
\mathcal{C}_{\mathrm{ss} 1}=\frac{p_{\mathrm{s}} \gamma_{\mathrm{s}} \mathcal{C}_{x}}{p_{\mathrm{s}} \gamma_{\mathrm{s}}+1}, \quad \mathcal{C}_{\mathrm{ss} 2}=\frac{p_{\mathrm{s}} \gamma_{\mathrm{s}} \mathcal{C}_{x}}{p_{\mathrm{s}} \gamma_{\mathrm{s}}+p_{\mathrm{p}} \mathcal{I}_{\mathrm{p}}+1},
$$

where $\mathcal{C}_{x}$ is the circularity coefficient of the cognitive user's signal.

We can rewrite the secondary achievable rate, after some manipulations as,

$$
\begin{aligned}
R_{\mathrm{s}}\left(p_{\mathrm{s}}, \mathcal{C}_{x}\right) & =A \log _{2}\left(p_{\mathrm{s}}^{2} \gamma_{\mathrm{s}}^{2}\left(1-\mathcal{C}_{x}^{2}\right)+2 p_{\mathrm{s}} \gamma_{\mathrm{s}}+1\right) \\
& +B \log _{2}\left(\frac{p_{\mathrm{s}}{ }^{2} \gamma_{\mathrm{s}}^{2}\left(1-\mathcal{C}_{x}^{2}\right)}{\left(1+p_{\mathrm{p}} \mathcal{I}_{\mathrm{p}}\right)^{2}}+\frac{2 p_{\mathrm{s}} \gamma_{\mathrm{s}}}{1+p_{\mathrm{p}} \mathcal{I}_{\mathrm{p}}}+1\right)
\end{aligned}
$$

where $A$ and $B$ are expressed, respectively, as,

$$
A=\frac{T-\tau}{2 T} p\left(1-P_{\mathrm{fa}}\right), \quad B=\frac{T-\tau}{2 T}(1-p)\left(1-P_{\mathrm{d}}\right) .
$$

On the other hand, the achievable rate of the PU depends on the probability of detection, according to the two following possible events: The first event occurs if the SU detects properly the activity of the PU with a probability $P_{\mathrm{d}}$, resulting in an interference-free communication at the PU side. The second event occurs with a probability $\left(1-P_{\mathrm{d}}\right)$ if the $\mathrm{SU}$ makes a wrong detection causing interference to the PU. Therefore, the achievable rate is defined as,

$$
R_{\mathrm{p}}\left(p_{\mathrm{s}}, \mathcal{C}_{x}\right)=P_{\mathrm{d}} R_{\mathrm{p}_{1}}+\left(1-P_{\mathrm{d}}\right) R_{\mathrm{p}_{2}},
$$

where $R_{\mathrm{p}_{1}}$ and $R_{\mathrm{p}_{2}}$ are the achievable rates in the two events and are defined as [15],

$$
\begin{aligned}
& R_{\mathrm{p}_{1}}=\log _{2}\left(1+p_{\mathrm{p}} \gamma_{\mathrm{p}}\right) \\
& R_{\mathrm{p}_{2}}=\log _{2}\left(1+\frac{p_{\mathrm{p}} \gamma_{\mathrm{p}}}{1+p_{\mathrm{s}} \mathcal{I}_{\mathrm{s}}}\right)+\frac{1}{2} \log _{2}\left(\frac{1-\mathcal{C}_{\mathrm{pp}}^{2}}{1-\mathcal{C}_{\mathrm{pi}}^{2}}\right),
\end{aligned}
$$

where $\mathcal{C}_{\mathrm{pp}}$ and $\mathcal{C}_{\mathrm{pi}}$ denote the circularity coefficients of the PU received signal from the direct link and the interference link, respectively, which can be expressed using Definition 2 as,

$$
\mathcal{C}_{\mathrm{pp}}=\frac{p_{\mathrm{s}} \mathcal{I}_{\mathrm{s}} \mathcal{C}_{x}}{p_{\mathrm{s}} \mathcal{I}_{\mathrm{s}}+p_{\mathrm{p}} \gamma_{\mathrm{p}}+1}, \quad \mathcal{C}_{\mathrm{pi}}=\frac{p_{\mathrm{s}} \mathcal{I}_{\mathrm{s}} \mathcal{C}_{x}}{p_{\mathrm{s}} \mathcal{I}_{\mathrm{s}}+1}
$$

After some manipulations, $R_{\mathrm{p}_{2}}$ reduces to,

$$
R_{\mathrm{p}_{2}}=\frac{1}{2} \log _{2}\left(1+\frac{2\left(p_{\mathrm{s}} \mathcal{I}_{\mathrm{s}}+1\right) p_{\mathrm{p}} \gamma_{\mathrm{p}}+p_{\mathrm{p}}^{2} \gamma_{\mathrm{p}}^{2}}{p_{\mathrm{s}}^{2} \mathcal{I}_{\mathrm{s}}^{2}\left(1-\mathcal{C}_{x}^{2}\right)+2 p_{\mathrm{s}} \mathcal{I}_{\mathrm{s}}+1}\right) .
$$

If we assume the $\mathrm{SU}$ is using PGS, the achievable rate of the $\mathrm{SU}$, considering $\mathcal{C}_{x}=0$ is,

$$
\begin{aligned}
R_{\mathrm{s}}\left(p_{\mathrm{s}}, 0\right) & =\frac{T-\tau}{T}\left(p\left(1-P_{\mathrm{fa}}\right) \log _{2}\left(1+p_{\mathrm{s}} \gamma_{\mathrm{s}}\right)\right. \\
& \left.+(1-p)\left(1-P_{\mathrm{d}}\right) \log _{2}\left(1+\frac{p_{\mathrm{s}} \gamma_{\mathrm{s}}}{1+p_{\mathrm{p}} \mathcal{I}_{\mathrm{p}}}\right)\right) .
\end{aligned}
$$


Similarly, the PU achievable rate reduces to,

$$
R_{\mathrm{p}}\left(p_{\mathrm{s}}, 0\right)=P_{\mathrm{d}} R_{\mathrm{p}_{1}}+\left(1-P_{\mathrm{d}}\right) \log _{2}\left(1+\frac{p_{\mathrm{p}} \gamma_{\mathrm{p}}}{1+p_{\mathrm{s}} \mathcal{I}_{\mathrm{s}}}\right) .
$$

\section{B. Outage Probability Performance}

In this section, we derive the expressions of the PU outage probability in the case of partial-CSI knowledge, when the SU employs PGS and IGS schemes, as a function of the SU transmit power and circularity coefficient.

1) Outage probability of the primary user in the proper signaling scenario: As per the full-CSI knowledge case, the outage probability expression of the PU will depend on the probability of detection. In the following, we derive the outage probability of the primary transmission where interweave cognitive radio uses PGS scheme for the secondary transmission. This probability is defined as,

$$
P_{\text {out }, \mathrm{p}}\left(p_{\mathrm{s}}, 0\right)=P_{\mathrm{d}} P_{1}+\left(1-P_{\mathrm{d}}\right) P_{2}\left(p_{\mathrm{s}}, 0\right),
$$

where $P_{1}$ is the PU outage probability in a free-interference link, i.e. with a correct detection in the SU. The probability $P_{1}$ is then expressed as follows,

$$
P_{1}=\operatorname{Pr}\left(\log _{2}\left(1+p_{\mathrm{p}} \gamma_{\mathrm{p}}\right)<R_{0}\right)=1-\exp \left(-\frac{\mu_{0}}{p_{\mathrm{p}} \bar{\gamma}_{\mathrm{p}}}\right),
$$

where $\mu_{0}=2^{R_{0}}-1$ and the random variable is $\gamma_{\mathrm{p}} \sim \operatorname{Exp}\left(\bar{\gamma}_{\mathrm{p}}\right)$. The outage probability of the PU, in the case of detection errors, is defined as,

$$
P_{2}\left(p_{\mathrm{s}}, 0\right)=\operatorname{Pr}\left(\log _{2}\left(1+\frac{p_{\mathrm{p}} \gamma_{\mathrm{p}}}{1+p_{\mathrm{s}} \mathcal{I}_{\mathrm{s}}}\right)<R_{0}\right) .
$$

By solving the inequality in (16), we can show that the PU outage probability in the case of wrong detection, conditioned on $\mathcal{I}_{\mathrm{s}}$, is given by,

$$
\begin{aligned}
P_{2}\left(p_{\mathrm{s}}, 0\right) \mid \mathcal{I}_{\mathrm{s}} & =\int_{0}^{\gamma_{0}} \frac{1}{\bar{\gamma}_{\mathrm{p}}} \exp \left(-\frac{y}{\bar{\gamma}_{\mathrm{p}}}\right) d y \\
& =1-\exp \left(\frac{\mu_{0}\left(1+p_{\mathrm{s}} \mathcal{I}_{\mathrm{s}}\right)}{p_{\mathrm{p}} \bar{\gamma}_{\mathrm{p}}}\right)
\end{aligned}
$$

where $\gamma_{0}=\frac{\mu_{0}}{p_{\mathrm{p}}}\left(1+p_{\mathrm{s}} \mathcal{I}_{\mathrm{s}}\right)$ represents the upper bound of the range of solutions in the inequality in (16). By averaging (17) over the exponential statistics of $\mathcal{I}_{\mathrm{s}}$, we obtain,

$$
\begin{aligned}
& P_{2}\left(p_{\mathrm{s}}, 0\right)=\mathbb{E}_{\mathcal{I}_{\mathrm{s}}}\left\{P_{2}\left(p_{\mathrm{s}}, 0\right) \mid \mathcal{I}_{\mathrm{s}}\right\} \\
& =1-\frac{1}{1+\frac{\mu_{0} p_{\mathrm{s}} \overline{\mathcal{I}}_{\mathrm{s}}}{p_{\mathrm{p}} \bar{\gamma}_{\mathrm{p}}}} \exp \left(-\frac{\mu_{0}}{p_{\mathrm{p}} \bar{\gamma}_{\mathrm{p}}}\right) \text {. }
\end{aligned}
$$

We can infer that the PU outage probability is an increasing function in $p_{\mathrm{s}}$ and $\overline{\mathcal{I}}_{\mathrm{s}}$. Thus, we can adjust the $\mathrm{SU}$ transmit power to control its impact on the PU performance.

2) Outage probability of the primary user in the improper signaling scenario: In the improper signaling scheme, the circularity coefficient is not zero and the outage probability expression will be as follows,

$$
P_{\text {out }, \mathrm{p}}\left(p_{\mathrm{s}}, \mathcal{C}_{x}\right)=P_{\mathrm{d}} P_{1}+\left(1-P_{\mathrm{d}}\right) P_{2}\left(p_{\mathrm{s}}, \mathcal{C}_{x}\right),
$$

where $P_{2}\left(p_{\mathrm{s}}, \mathcal{C}_{x}\right)=\operatorname{Pr}\left(R_{\mathrm{p}_{2}}<R_{0}\right)$ and can be rewritten equivalently as,

$$
P_{2}\left(p_{\mathrm{s}}, \mathcal{C}_{x}\right)=\operatorname{Pr}\left(g\left(\gamma_{\mathrm{p}}, \mathcal{I}_{\mathrm{s}}\right)<0\right),
$$

where $g\left(\gamma_{\mathrm{p}}, \mathcal{I}_{\mathrm{s}}\right)=p_{\mathrm{p}}^{2} \gamma_{\mathrm{p}}^{2}+2\left(p_{\mathrm{s}} \mathcal{I}_{\mathrm{s}}+1\right) p_{\mathrm{p}} \gamma_{\mathrm{p}}-\left(2^{2 R_{0}}-\right.$ 1) $\left(p_{\mathrm{s}}^{2} \mathcal{I}_{\mathrm{s}}^{2}\left(1-\mathcal{C}_{x}{ }^{2}\right)+2 p_{\mathrm{s}} \mathcal{I}_{\mathrm{s}}+1\right)$. After solving the quadratic inequality in (20), we can state that,

$$
g\left(\gamma_{\mathrm{p}}, \mathcal{I}_{\mathrm{s}}\right)<0 \Leftrightarrow \gamma_{p_{1}}\left(\mathcal{I}_{\mathrm{s}}\right)<\gamma_{\mathrm{p}}<\gamma_{p_{2}}\left(\mathcal{I}_{\mathrm{s}}\right) .
$$

Since $\gamma_{p_{1}}<0$ then, $0<\gamma_{\mathrm{p}}<\gamma_{p_{2}}\left(\mathcal{I}_{\mathrm{s}}\right)$, where, $\gamma_{p_{2}}(x)=\frac{2^{R_{0}} \sqrt{a\left(\mathcal{C}_{x}\right) p_{\mathrm{s}}^{2} x^{2}+2 p_{\mathrm{s}} x+1}-\left(p_{\mathrm{s}} x+1\right)}{p_{\mathrm{p}}}$ and, $a\left(\mathcal{C}_{x}\right)=$ $\frac{1}{2^{2 R_{0}}}+\left(1-\frac{1}{2^{2 R_{0}}}\right)\left(1-\mathcal{C}_{x}^{2}\right)$. We can infer that the outage happens for a specific range of $\gamma_{p}$, which is an expected result. Then,

$$
\begin{aligned}
P_{2}\left(p_{\mathrm{s}}, \mathcal{C}_{x}\right) \mid \mathcal{I}_{\mathrm{s}} & =\int_{0}^{\gamma_{p_{2}}\left(\mathcal{I}_{\mathrm{s}}\right)} \frac{1}{\bar{\gamma}_{\mathrm{p}}} \exp \left(-\frac{y}{\bar{\gamma}_{\mathrm{p}}}\right) d y \\
& =1-\exp \left(-\frac{\gamma_{p_{2}}\left(\mathcal{I}_{\mathrm{s}}\right)}{\bar{\gamma}_{\mathrm{p}}}\right) .
\end{aligned}
$$

By averaging (22) over the statistics of $\mathcal{I}_{\mathrm{s}}$, we obtain,

$$
\begin{gathered}
P_{2}\left(p_{\mathrm{s}}, \mathcal{C}_{x}\right)=\mathbb{E}_{\mathcal{I}_{\mathrm{s}}}\left\{P_{2}\left(p_{\mathrm{s}}, \mathcal{C}_{x}\right) \mid \mathcal{I}_{\mathrm{s}}\right\} \\
P_{2}\left(p_{\mathrm{s}}, \mathcal{C}_{x}\right)=1-\int_{0}^{\infty} \frac{1}{\overline{\mathcal{I}}_{\mathrm{s}}} \exp \left(-\frac{x}{\overline{\mathcal{I}}_{\mathrm{s}}}\right) \exp \left(-\frac{\gamma_{p_{2}}(x)}{\bar{\gamma}_{\mathrm{p}}}\right) d x .
\end{gathered}
$$

Unfortunately, there is no closed form expression for the integral in (24). However, we can limit the outage probability by an upper bound to guarantee an acceptable minimum outage probability performance for the PU. In this context, we find an upper bound to the PU's outage probability by using Jensen's inequality as in [32], [37], which states that for a random variable $X$ and a convex function $\omega$ we have,

$$
\omega(E(X)) \leq E(\omega(X)) .
$$

In our case, we consider the following function: $\omega(x)=$ $\exp \left(\frac{-2^{R_{0}} \sqrt{a\left(\mathcal{C}_{x}\right) p_{\mathrm{s}}^{2} x^{2}+2 p_{\mathrm{s}} x+1}+p_{\mathrm{s}} x+1}{p_{\mathrm{p}} \gamma_{\mathrm{p}}}\right)$ where $\omega$ is convex (See Appendix A for the proof). Then, we can use the Jensen's inequality and find an upper bound for $P_{2}\left(p_{\mathrm{s}}, \mathcal{C}_{x}\right)$, expressed as,

$$
P_{2}^{\mathrm{UP}}\left(p_{\mathrm{s}}, \mathcal{C}_{x}\right)=1-\exp \left(-\frac{\gamma_{p_{2}}\left(\overline{\mathcal{I}}_{\mathrm{s}}\right)}{\bar{\gamma}_{\mathrm{p}}}\right) .
$$

As a first inspection from this result, the upper bound probability for a proper signal $\left(\mathcal{C}_{x}=0\right)$ is different from the exact probability expression calculated in the previous section, i.e. $P_{\text {out }, \mathrm{p}}^{\mathrm{UP}}\left(p_{\mathrm{s}}, 0\right) \neq P_{\mathrm{out}, \mathrm{p}}\left(p_{\mathrm{s}}, 0\right)$. Thus, when designing our system, to have the maximum gain, we will consider the solution that maximizes the SU achievable rate between the improper upper bound based design and the proper exact one, and therefore, we will have an opportunistic system that achieves maximum gain.

\section{Perfect CSI Based Design}

In this section, we design the interweave cognitive radio system considering a perfect instantaneous CSI of all the considered links at the SU side. To this end, we optimize the SU operation by maximizing its achievable rate while keeping a minimum achievable rate for the primary communication. The optimization parameters are the transmitted power $p_{\mathrm{s}}$ and the circularity coefficient $\mathcal{C}_{x}$. 


\section{A. Proper Gaussian Signaling Design}

First, we design the interweave cognitive radio assuming PGS scheme in order to be used as a reference for our proposed IGS scheme. The PGS design problem is expressed as follows,

$$
\begin{array}{cl}
\max _{p_{\mathrm{s}}} & R_{\mathrm{s}}\left(p_{\mathrm{s}}, 0\right) \\
\text { subject to } & R_{\mathrm{p}}\left(p_{\mathrm{s}}, 0\right) \geq R_{\min } \\
& 0 \leq p_{\mathrm{s}} \leq p_{\mathrm{s}, \max } .
\end{array}
$$

Before solving the design problem, the following theorem gives the feasibility conditions for the interweave cognitive radio system's operation and presents the system's parameters of the optimal design.

Theorem 1. In an interweave cognitive radio system with $P G S$ scheme adopted at both the primary and secondary networks, and considering an instantaneous CSI knowledge in the $S U$ side, the feasibility condition and the optimal $S U$ power needed to maximize the achievable rate while allowing the PU operation with a minimum rate of $R_{\min }$, are found based on problem (26), respectively, as follows,

- $\gamma_{\mathrm{p}} \geq \gamma_{\mathrm{th}} \triangleq \frac{2^{R_{\min }-1}}{p_{\mathrm{p}}}$, which confirms the ability of the $P U$ direct link to support its requirements. ${ }^{2}$.

- The optimal SU power is found to be,

$$
P_{\mathrm{s}, \text { opt }}= \begin{cases}p_{\mathrm{s}, \max } & P_{\mathrm{d}} \geq P_{\mathrm{d}_{1}} \text { or } \quad \mathcal{I}_{\mathrm{s}} \leq \mathcal{I}_{\mathrm{s}, \mathrm{PGS}} \\ \max \left(\hat{p}_{\mathrm{s}_{1}}, 0\right) & \text { otherwise }\end{cases}
$$

where

$$
\begin{aligned}
P_{\mathrm{d}_{1}} & =\frac{R_{\min }}{R_{\mathrm{p}_{1}}} \\
\mathcal{I}_{\mathrm{s}, \mathrm{PGS}} & =\frac{1}{p_{\mathrm{s}, \max }}\left(\frac{p_{\mathrm{p}} \gamma_{\mathrm{p}}}{2^{\frac{R_{\mathrm{min}}-P_{\mathrm{d}} R_{\mathrm{p}_{1}}}{1-P_{\mathrm{d}}}}-1}-1\right) \\
\hat{p}_{\mathrm{s}_{1}} & =\frac{1}{\mathcal{I}_{\mathrm{s}}}\left(\frac{p_{\mathrm{p}} \gamma_{\mathrm{p}}}{2^{\frac{R_{\min }-P_{\mathrm{d}} R_{\mathrm{p}_{1}}}{1-P_{\mathrm{d}}}}-1}-1\right) .
\end{aligned}
$$

Proof. To solve (26), we observe first, that the objective function, i.e., $R_{\mathrm{s}}\left(p_{\mathrm{s}}, 0\right)$, is strictly increasing with $p_{\mathrm{s}}$, as it can be easily proved. Therefore, the solution of the problem (26) reduces to the maximum power that satisfies its constraints. Now, we need to study first the rate constraint in (26) that can be rewritten equivalently as,

$$
\frac{p_{\mathrm{p}} \gamma_{\mathrm{p}}}{1+p_{\mathrm{s}} \mathcal{I}_{\mathrm{s}}} \geq z
$$

where $z=2^{\frac{R_{\min }-P_{\mathrm{d}} R_{\mathrm{p}_{1}}}{1-P_{\mathrm{d}}}}-1$. One can observe that $z$ can be either positive or negative, giving the following two possible cases:

i. $z<0$ is equivalent to $P_{\mathrm{d}}>\frac{R_{\min }}{R_{\mathrm{p}_{1}}} \triangleq P_{\mathrm{d}_{1}}$. In this case, the constraint in (28) is always valid and hence, the solution reduces to $P_{\mathrm{s}, \mathrm{opt}}=p_{\mathrm{s}, \max }$.

ii. $z \geq 0$ is equivalent to $P_{\mathrm{d}} \leq P_{\mathrm{d}_{1}}$, allowing us to rewrite the constraint in (28), as $p_{\mathrm{s}} \leq \hat{p}_{\mathrm{s}_{1}}$. In this case, the solution becomes $\min \left(p_{\mathrm{s}, \max }, \tilde{p}_{\mathrm{s}}\right)$, which captures the following

\footnotetext{
${ }^{2}$ The gain of the PU direct link determines whether or not its quality is capable of supporting the PU requirements. The PU rate should satisfy $R_{\mathrm{p}}(0,0)>R_{\min }$ and subsequently is reduced to $R_{\mathrm{p}_{1}}>R_{\min }$, where $R_{\mathrm{p}_{1}}$ is defined in 8 .
}

scenarios:

Case II-a: $P_{\mathrm{s}, \mathrm{opt}}=p_{\mathrm{s}, \max }$, when $\hat{p}_{\mathrm{s}_{1}} \geq p_{\mathrm{s}, \max }$ that is equivalent to $\mathcal{I}_{\mathrm{s}} \leq \mathcal{I}_{\mathrm{s}, \mathrm{PGS}}$.

Case II-b: $P_{\mathrm{s}, \text { opt }}=\tilde{p}_{\mathrm{s}}$, when $\hat{p}_{\mathrm{s}_{1}}<p_{\mathrm{s}, \max }$ that is equivalent to $\mathcal{I}_{\mathrm{s}}>\mathcal{I}_{\mathrm{s}, \mathrm{PGS}}$.

These results show that the SU operation is conditioned by its detection performances and its interference to the PU activity. Therefore, the SU may operate with its full transmit power either in an accurate detection system or by guaranteeing a maximum predefined threshold of interference to the PU.

\section{B. Improper Gaussian Signaling Design}

In this section, we optimize the IGS signal parameters $\left(p_{\mathrm{s}}, \mathcal{C}_{x}\right)$ in order to improve the $\mathrm{SU}$ operation performance while maintaining the $\mathrm{PU}$ minimum rate requirement. To this end, the next considered optimization problem is,

$$
\begin{array}{cl}
\max _{p_{\mathrm{s}}, \mathcal{C}_{x}} & R_{\mathrm{s}}\left(p_{\mathrm{s}}, \mathcal{C}_{x}\right) \\
\text { subject to } & R_{\mathrm{p}}\left(p_{\mathrm{s}}, \mathcal{C}_{x}\right) \geq R_{\min } \\
& 0 \leq p_{\mathrm{s}} \leq p_{\mathrm{s}, \max } \\
& 0 \leq \mathcal{C}_{x} \leq 1 .
\end{array}
$$

After some manipulations, the first constraint in (29) can be rewritten as,

$$
y\left(p_{\mathrm{s}}, \mathcal{C}_{x}\right)=p_{\mathbf{s}}^{2} \mathcal{I}_{\mathbf{s}}^{2}\left(1-\mathcal{C}_{x}^{2}\right) \psi(0)+2 p_{\mathrm{s}} \mathcal{I}_{\mathbf{s}} \psi(1)+\psi(2) \geq 0,
$$

where $\psi(\zeta)$ is defined as,

$$
\psi(\zeta)=2^{\zeta R_{\mathrm{p}_{1}}}-2^{2 \frac{R_{\mathrm{min}}-P_{\mathrm{d}} R_{\mathrm{p}_{1}}}{1-P_{\mathrm{d}}}} .
$$

The behavior of (30) is governed by the sign of $\psi(0), \psi(1)$ and $\psi(2)$. Thus, we consider the following cases:

Case 1: when $\psi(0) \geq 0$, i.e. $P_{\mathrm{d}} \geq P_{\mathrm{d}_{1}}$ then $\psi(1)$ and $\psi(2)>$ 0 since $\psi(\zeta)$ is increasing. Thus, $y\left(p_{\mathrm{s}}, \mathcal{C}_{x}\right)>0, \forall p_{\mathrm{s}}$ and $\forall \mathcal{C}_{x}$. As $R_{\mathrm{s}}\left(p_{\mathrm{s}}, \mathcal{C}_{x}\right)$ is increasing in $p_{\mathrm{s}}$ and decreasing in $\mathcal{C}_{x}$, the IGS solution reduces to $\left(P_{\mathrm{s}, \mathrm{opt}}, \mathcal{C}_{\mathrm{x}, \mathrm{opt}}\right)=\left(p_{\mathrm{s}, \max }, 0\right)$.

Case 2: when $\psi(0)<0$, i.e. $P_{\mathrm{d}}<P_{\mathrm{d}_{1}}$ one can prove that $\psi(2)>0^{3}$. Thus, $y\left(p_{\mathrm{s}}, \mathcal{C}_{x}\right)=0$ has one positive and one negative root. Therefore, the power is upper-bounded by the positive root $\hat{p}_{\mathrm{s}}\left(\mathcal{C}_{x}\right)$ that has the following expression,

$$
\hat{p}_{\mathrm{s}}\left(\mathcal{C}_{x}\right)=\frac{\sqrt{\psi(1)^{2}-\left(1-\mathcal{C}_{x}^{2}\right) \psi(0) \psi(2)}+\psi(1)}{-\left(1-\mathcal{C}_{x}^{2}\right) \psi(0) \mathcal{I}_{\mathrm{s}}} .
$$

Thus, the optimization problem in (29) becomes,

$$
\begin{array}{cl}
\max _{p_{\mathrm{s}}, \mathcal{C}_{x}} & R_{\mathrm{s}}\left(p_{\mathrm{s}}, \mathcal{C}_{x}\right) \\
\text { subject to } & 0 \leq p_{\mathrm{s}} \leq \hat{\mathcal{F}}\left(\mathcal{C}_{x}\right) \\
& 0 \leq \mathcal{C}_{x} \leq 1,
\end{array}
$$

where $\mathcal{F}\left(\mathcal{C}_{x}\right)=\min \left(p_{\mathrm{s}, \max }, \hat{p}_{\mathrm{s}}\left(\mathcal{C}_{x}\right)\right)$.

As mentioned before, $R_{\mathrm{s}}\left(p_{\mathrm{s}}, \mathcal{C}_{x}\right)$ is increasing in $p_{\mathrm{s}}$ and decreasing in $\mathcal{C}_{x}$. Moreover, one can easily prove that $\hat{p}_{\mathrm{s}}\left(\mathcal{C}_{x}\right)$ is increasing in $\mathcal{C}_{x}$ over the considered interval. Consequently, we

\footnotetext{
${ }^{3}$ Since $R_{\mathrm{p}_{1}}>R_{\mathrm{min}}$, then $\psi(2)>0$.
} 
can derive the optimal signal design according to the following theorem.

Theorem 2. In a feasible interweave cognitive radio system with IGS scheme adopted at the primary network, a PGS scheme at the secondary network and an instantaneous CSI knowledge in the $S U$ side, when the probability of detection is below a predefined threshold $P_{\mathrm{d}_{1}}$, the optimal $S U$ transmit power and circularity coefficient will be set depending on the conditions stated in equation (34), where,

$$
\begin{aligned}
\mathcal{I}_{\mathrm{s}_{1}} & =\max \left(\min \left(\frac{\psi(1) \gamma_{\mathrm{s}}}{\psi(0)}, \frac{\psi(1) \gamma_{\mathrm{s}}}{\psi(0)\left(1+p_{\mathrm{p}} I_{\mathrm{p}}\right)}\right), 0\right) \\
\mathcal{I}_{\mathrm{s}_{2}} & =\max \left(\max \left(\frac{\psi(1) \gamma_{\mathrm{s}}}{\psi(0)}, \frac{\psi(1) \gamma_{\mathrm{s}}}{\psi(0)\left(1+p_{\mathrm{p}} I_{\mathrm{p}}\right)}\right), 0\right) \\
\mathcal{C}_{x}^{*} & =\sqrt{1+\frac{\psi(2)+2 \psi(1) p_{\mathrm{s}, \max } \overline{\mathcal{I}}_{\mathrm{s}}}{p_{\mathrm{s}, \max }^{2} \overline{\mathcal{I}}_{\mathrm{s}}^{2} \psi(0)}} \\
\mathcal{C}_{x_{1}} & =\arg \max \left(R_{\mathrm{s}}(0), R_{\mathrm{s}}\left(\mathcal{C}_{x}^{* *}\right), \lim _{\mathcal{C}_{x} \rightarrow 1} R_{\mathrm{s}}\left(\mathcal{C}_{x}\right)\right) \\
\mathcal{C}_{x_{2}} & =\arg \max \left(R_{\mathrm{s}}(0), R_{\mathrm{s}}\left(\mathcal{C}_{x}^{* *}\right), R_{\mathrm{s}}\left(\mathcal{C}_{x}^{*}\right)\right) .
\end{aligned}
$$

Proof. The three following cases detail the relation between $p_{\mathrm{s}, \max }$ and $\hat{p}_{\mathrm{s}}\left(\mathcal{C}_{x}\right)$ since the solution of the problem (33) depends on this relation.

Case 1: $\mathcal{F}\left(\mathcal{C}_{x}\right)=p_{\mathrm{s}, \max }$, which occurs when $\hat{p}_{\mathrm{s}}(0)>$ $p_{\mathrm{s}, \max } \Leftrightarrow \mathcal{I}_{\mathrm{s}}<\mathcal{I}_{\mathrm{s}_{1}}$. Therefore, (33) reduces to,

$$
\begin{array}{cl}
\max _{\mathcal{C}_{x}} & R_{\mathrm{s}}\left(p_{\mathrm{s}, \max }, \mathcal{C}_{x}\right) \\
\text { subject to } & 0 \leq \mathcal{C}_{x} \leq 1 .
\end{array}
$$

Since $R_{\mathrm{s}}\left(p_{\mathrm{s}, \max }, \mathcal{C}_{x}\right)$ is decreasing in $\mathcal{C}_{x}$, the solution reduces to the proper one, i.e., $\left(P_{\mathrm{s}, \text { opt }}, \mathcal{C}_{\mathrm{x}, \text { opt }}\right)=\left(p_{\mathrm{s}, \max }, 0\right)$.

Case 2: $\mathcal{F}\left(\mathcal{C}_{x}\right)=\hat{p}_{\mathrm{s}}\left(\mathcal{C}_{x}\right)$, which occurs when $\lim _{\mathcal{C}_{x} \rightarrow 1} \hat{p}_{\mathrm{s}}\left(\mathcal{C}_{x}\right)<p_{\mathrm{s}, \max }$. This limit is equal to $-\frac{\psi(2)}{2 \mathcal{I}_{\mathrm{s}} \psi(1)}$ if $\psi(1)<0$ and to positive infinity otherwise. Then, this case will not be valid only if this limit is below $p_{\mathrm{s}, \max }$. Therefore, (33) reduces to,

$$
\begin{array}{cl}
\max _{\mathcal{C}_{x}} & R_{\mathrm{s}}\left(\hat{p}_{\mathrm{s}}\left(\mathcal{C}_{x}\right), \mathcal{C}_{x}\right) \triangleq R_{\mathrm{S}}\left(\mathcal{C}_{x}\right) \\
\text { subject to } & 0 \leq \mathcal{C}_{x} \leq 1,
\end{array}
$$

where $R_{\mathrm{s}}\left(\mathcal{C}_{x}\right)$ can be rewritten as,

$$
R_{\mathrm{s}}\left(\mathcal{C}_{x}\right)=A \log _{2}\left(\hat{a}_{1} \hat{p}_{\mathrm{s}}\left(\mathcal{C}_{x}\right)+\hat{a}_{2}\right)+B \log _{2}\left(\hat{b}_{1} \hat{p}_{\mathrm{s}}\left(\mathcal{C}_{x}\right)+\hat{b}_{2}\right),
$$

where, $\hat{a}_{1}=2 \gamma_{\mathrm{s}}-\frac{2 \psi(1) \gamma_{\mathrm{s}}^{2}}{\psi(0) \mathcal{I}_{\mathrm{s}}}, \hat{a}_{2}=1-\frac{\psi(2) \gamma_{\mathrm{s}}^{2}}{\psi(0) \mathcal{I}_{\mathrm{s}}^{2}}, \hat{b}_{1}=\frac{2 \gamma_{\mathrm{s}}}{1+p_{\mathrm{p}} \mathcal{I}_{\mathrm{p}}}-$ $\frac{2 \psi(1) \gamma_{\mathrm{s}}^{2}}{\psi(0) \mathcal{I}_{\mathrm{s}}\left(1+p_{\mathrm{p}} \mathcal{I}_{\mathrm{p}}\right)^{2}}, \hat{b}_{2}=1-\frac{\psi(2) \gamma_{\mathrm{s}}^{2}}{\psi(0) \mathcal{I}_{\mathrm{s}}^{2}\left(1+p_{\mathrm{p}} \mathcal{I}_{\mathrm{p}}\right)^{2}},($ See Appendix B for the proof). The behavior of $R_{\mathrm{s}}\left(\mathcal{C}_{x}\right)$ depends on the sign of $\hat{a}_{1}$ and $\hat{b}_{1}$ giving the following possible cases:

Case 2-a: $\hat{a}_{1}>0$ and $\hat{b}_{1}>0 \Leftrightarrow \mathcal{I}_{\mathrm{s}}>\mathcal{I}_{1}=\frac{\psi(1) \gamma_{\mathrm{s}}}{\psi(0)}$ and $\mathcal{I}_{\mathrm{s}}>\mathcal{I}_{2}=\frac{\psi(1) \gamma_{\mathrm{s}}}{\psi(0)\left(1+p_{\mathrm{p}} I_{\mathrm{p}}\right)}$ yielding increasing characteristics of $R_{\mathrm{s}}\left(\mathcal{C}_{x}\right)$ in $\mathcal{C}_{x}$. Thus, the solution reduces to $\left(P_{\mathrm{s}, \text { opt }}, \mathcal{C}_{\mathrm{x}, \text { opt }}\right)=$ $\left(\lim _{\mathcal{C}_{x} \rightarrow 1} \hat{p}_{\mathrm{s}}\left(\mathcal{C}_{x}\right), 1\right)$.

Case 2-b: $\hat{a}_{1} \leq 0$ and $\hat{b}_{1} \leq 0 \Leftrightarrow \mathcal{I}_{\mathrm{s}} \leq \mathcal{I}_{1}$ and $\mathcal{I}_{\mathrm{s}} \leq \mathcal{I}_{2}$ yielding decreasing characteristics of $R_{\mathrm{s}}\left(\mathcal{C}_{x}\right)$ in $\mathcal{C}_{x}$. Thus, the solution reduces to $\left(P_{\mathrm{s}, \text { opt }}, \mathcal{C}_{\mathrm{x}, \text { opt }}\right)=\left(\hat{p}_{\mathrm{s}}(0), 0\right)$

Case 2-c: $\hat{a}_{1} \hat{b}_{1}<0$ yielding unknown characteristics of $R_{\mathrm{S}}\left(\mathcal{C}_{x}\right)$ in $\mathcal{C}_{x}$. Therefore, the maximum performance of $R_{\mathrm{S}}\left(\mathcal{C}_{x}\right)$ occurs either at one of the bounds $\mathcal{C}_{x}$ bound or at a stationary point. To investigate the stationary points of $R_{\mathrm{s}}\left(\mathcal{C}_{x}\right)$, we find its derivative with respect to $\mathcal{C}_{x}$ and equate it to zero, obtaining one stationary point that is written as,

$$
\hat{p}_{\mathrm{ss}}=-\frac{\hat{a}_{1} \hat{b}_{2} A+\hat{b}_{1} \hat{a}_{2} B}{\hat{a}_{1} \hat{b}_{1}(A+B)} .
$$

This stationary point is considered only if $\hat{p}_{\mathrm{ss}} \in$ $\left[\hat{p}_{\mathrm{s}}(0), \lim _{\mathcal{C}_{x} \rightarrow 1} \hat{p}_{\mathrm{s}}\left(\mathcal{C}_{x}\right)\right]$. In this case, the corresponding $\mathcal{C}_{x}$ is $\mathcal{C}_{x}^{* *}=\mathcal{G}\left(p_{\mathrm{ss}}\right)$, where the function $\mathcal{G}\left(p_{\mathrm{s}}\right)=$ $\sqrt{1-\frac{\psi(2)+2 \psi(1) p_{\mathrm{s}} \mathcal{I}_{\mathrm{s}}}{-p_{\mathrm{s}}^{2} \mathcal{I}_{\mathrm{s}}^{2} \psi(0)}}$ is the inverse function of $\hat{p}_{\mathrm{s}}\left(\mathcal{C}_{x}\right)$. As a result, the solution in this case reduces to, $\mathcal{C}_{x, \text { opt }}=\mathcal{C}_{x_{1}}=$ $\arg \max \left(R_{\mathrm{S}}(0), R_{\mathrm{S}}\left(\mathcal{C}_{x}^{* *}\right), \lim _{\mathcal{C}_{x} \rightarrow 1} R_{\mathrm{S}}\left(\mathcal{C}_{x}\right)\right)$ and $P_{\mathrm{s}, \text { opt }}=\hat{p}_{\mathrm{s}}\left(\mathcal{C}_{x_{1}}\right)$.

Case 3: $\min \left(p_{\mathrm{s}, \max }, \hat{p}_{\mathrm{s}}\left(\mathcal{C}_{x}\right)\right)=\left\{\begin{array}{cc}\hat{p}_{\mathrm{s}}\left(\mathcal{C}_{x}\right) & 0 \leq \mathcal{C}_{x} \leq \mathcal{C}_{x}^{*} \\ p_{\mathrm{s}, \max } & \mathcal{C}_{x}^{*}<\mathcal{C}_{x} \leq 1,\end{array}\right.$ where $\mathcal{C}_{x}^{*}=\mathcal{G}\left(p_{\mathrm{s}, \max }\right)$ is the intersection point between $p_{\mathrm{s}, \max }$ and $\hat{p}_{\mathrm{s}}\left(\mathcal{C}_{x}\right)$. In this case, we solve the problem in each $\mathcal{C}_{x}$ interval and choose the solution that maximizes the objective function. The first optimization problem is defined over $0 \leq \mathcal{C}_{x} \leq \mathcal{C}_{x}^{*}$ as,

$$
\text { P1: } \begin{array}{ll}
\max _{\mathcal{C}_{x}} & R_{\mathrm{s}}\left(\mathcal{C}_{x}\right) \\
\text { subject to } & 0 \leq \mathcal{C}_{x} \leq \mathcal{C}_{x}^{*} .
\end{array}
$$

The solution of this problem is similar to Case 2 problem as:

- when $\hat{a}_{1}>0$ and $\hat{b}_{1}>0$, the solution reduces to $\left(P_{\mathrm{s}, \mathbf{P} 1}^{*}, \mathcal{C}_{\mathbf{P} 1}^{*}\right)=\left(\hat{p}_{\mathrm{s}}\left(\mathcal{C}_{x}^{*}\right), \mathcal{C}_{x}^{*}\right)$.

- when $\hat{a}_{1} \leq 0$ and $\hat{b}_{1} \leq 0$, the solution reduces to, $\left(P_{\mathrm{s}, \mathbf{P} 1}^{*}, \mathcal{C}_{\mathbf{P} \mathbf{1}}^{*}\right)=\left(\hat{p}_{\mathrm{s}}(0), 0\right)$.

- when $\hat{a}_{1} \hat{b}_{1}<0$, the solution reduces to, $\mathcal{C}_{\mathbf{P} 1}^{*}=$ $\arg \max \left(R_{\mathrm{s}}(0), R_{\mathrm{s}}\left(\mathcal{C}_{x}^{* *}\right), R_{\mathrm{s}}\left(\mathcal{C}_{x}^{*}\right)\right)$ and $P_{\mathrm{s}, \mathbf{P} \mathbf{1}}^{*}=\hat{p}_{\mathrm{s}}\left(\mathcal{C}_{\mathbf{P} \mathbf{1}}^{*}\right)$. where the stationary point is considered only if $p_{\mathrm{ss}} \in$ $\left[p_{\mathrm{s}}(0), \hat{p}_{\mathrm{s}}\left(\mathcal{C}_{x}^{*}\right)\right]$ or equivalently if $\mathcal{C}_{x}^{* *} \in\left[0, \mathcal{C}_{x}^{*}\right]$.

As for the second optimization problem over $\mathcal{C}_{x}^{*}<\mathcal{C}_{x} \leq 1$, it is defined as,

$$
\begin{aligned}
& \text { P2: } \max _{\mathcal{C}_{x}} \quad R_{\mathrm{s}}\left(p_{\mathrm{s}, \max }, \mathcal{C}_{x}\right) \\
& \text { subject to } \quad \mathcal{C}_{x}^{*} \leq \mathcal{C}_{x} \leq 1 \text {. }
\end{aligned}
$$


The solution reduces to $\left(P_{\mathrm{s}, \mathbf{P} \mathbf{2}}^{*}, \mathcal{C}_{\mathbf{P} \mathbf{2}}^{*}\right)=\left(p_{\mathrm{s}, \max }, \mathcal{C}_{x}^{*}\right)$, which is included as the second boundary in $\mathbf{P 1}$. As a result, the solution of both $\mathbf{P 1}$ and $\mathbf{P 2}$ is the solution of P1, i.e., $\left(P_{\mathrm{s}, \mathrm{opt}}, \mathcal{C}_{\mathrm{x}, \mathrm{opt}}\right)=$ $\left(P_{\mathrm{s}, \mathbf{P} 1}^{*}, \mathcal{C}_{\mathbf{P} 1}^{*}\right)$.

\section{Partial-CSi Based Design}

In this section, we design the interweave cognitive radio system in the general scenario of partially unknown CSI at the SU. The latter can estimate the average value of the PU direct channel and the interference channel from the SU to the PU receiver.

\section{A. Proper Gaussian Signaling With Partial CSI Based Design}

Now, since the PU direct link and the interference link from the SU to the PU are unknown, we will consider an outage probability constraint on the PU communication. In this case, we can achieve a PGS based design using Partial CSI, after solving the following optimization problem,

$$
\begin{array}{cc}
\max _{p_{\mathrm{s}}} & R_{\mathrm{s}}\left(p_{\mathrm{s}}, 0\right) \\
\text { subject to } & P_{\text {out }}\left(p_{\mathrm{s}}, 0\right) \leq P_{0} \\
& 0 \leq p_{\mathrm{s}} \leq p_{\mathrm{s}, \max } .
\end{array}
$$

First, we define the feasibility condition of interweave cognitive radio system with partial CSI. Then, we present the system's parameters of the optimal design, according to the following theorem.

Theorem 3. In an interweave cognitive radio system with $P G S$ scheme adopted at both the primary and secondary networks operating at a target rate $R_{0}$ and a maximum outage probability $P_{0}$ for the primary communication, assume the availability of $P U$ direct link and the interference link from the SU to the PU average CSI and the secondary links instantaneous CSI at the secondary transmitter (ST) side. The feasibility condition and the optimal SU power needed to maximize the achievable rate while allowing the PU operation with a minimum rate of $R_{\min }$ are found based on problem (39), respectively, as follows,

- $\bar{\gamma}_{\mathrm{p}}>\bar{\gamma}_{\mathrm{th}} \triangleq \frac{2^{R_{0}}-1}{-p_{\mathrm{p}} \log \left(1-P_{0}\right)}$, which confirms the ability of the $P U$ direct link to support its requirements. ${ }^{4}$

- The optimal SU power is found to be,

$$
P_{\mathrm{s}, \mathrm{opt}}= \begin{cases}p_{\mathrm{s}, \max } & P_{\mathrm{d}} \geq P_{\mathrm{d}_{1}} \quad \text { or } \quad \overline{\mathcal{I}}_{\mathrm{s}} \leq \overline{\mathcal{I}}_{\mathrm{s}_{1}} \\ \max \left(p_{\mathrm{s}_{1}}, 0\right) & \text { otherwise }\end{cases}
$$

where

$$
\begin{aligned}
P_{\mathrm{d}_{1}} & =\frac{1-P_{0}}{1-P_{1}} \\
\overline{\mathcal{I}}_{\mathrm{s}_{1}} & =\frac{p_{\mathrm{p}} \bar{\gamma}_{\mathrm{p}}}{p_{\mathrm{s}, \max } \mu_{0}}\left(\frac{\exp \left(-\frac{\mu_{0}}{p_{\mathrm{p}} \overline{\mathrm{p}}_{\mathrm{p}}}\right)}{1-\frac{P_{0}-P_{\mathrm{d}} P_{1}}{1-P_{\mathrm{d}}}}-1\right) \\
p_{\mathrm{s}_{1}} & =\frac{p_{\mathrm{p}} \bar{\gamma}_{\mathrm{p}}}{\overline{\mathcal{I}}_{\mathrm{s}} \mu_{0}}\left(\frac{\exp \left(-\frac{\mu_{0}}{p_{\mathrm{p}} \bar{\gamma}_{\mathrm{p}}}\right)}{1-\frac{P_{0}-P_{P_{1}}}{1-P_{\mathrm{d}}}}-1\right) .
\end{aligned}
$$

\footnotetext{
${ }^{4}$ The necessary condition depends on the quality of the PU direct link whether it is capable of supporting the PU requirements. The PU rate requirement of operation should satisfy $P_{\text {out,p }}(0,0)<P_{0}$ and subsequently is reduced to $\bar{\gamma}_{\mathrm{p}}>\bar{\gamma}_{\mathrm{th}} \triangleq \frac{2^{R_{0}}-1}{-p_{\mathrm{p}} \log \left(1-P_{0}\right)}$.
}

Proof. The constraint in (39) can be rewritten as,

$$
\frac{1}{1+\frac{\mu_{0} p_{\mathrm{s}} \overline{\mathcal{I}}_{\mathrm{s}}}{p_{\mathrm{p}} \bar{\gamma}_{\mathrm{p}}}} \exp \left(-\frac{\mu_{0}}{p_{\mathrm{p}} \bar{\gamma}_{\mathrm{p}}}\right) \geq 1-\frac{P_{0}-P_{\mathrm{d}} P_{1}}{1-P_{\mathrm{d}}} \triangleq \mathcal{X} .
$$

$\mathcal{X}>0 \Leftrightarrow P_{\mathrm{d}}<P_{\mathrm{d}_{1}}{ }^{5}$. Then, (41) becomes $p_{\mathrm{s}} \leq p_{\mathrm{s}_{1}}$. The optimal choice for the transmitted power will be $P_{\mathrm{s}, \mathrm{opt}}=$ $\min \left(p_{\mathrm{s}_{1}}, p_{\mathrm{s}, \max }\right)$. This value can be determined by a simple mathematical manipulation. In fact, $p_{\mathrm{s}_{1}}<p_{\mathrm{s}, \max }$ is equivalent to $\overline{\mathcal{I}}_{\mathrm{s}}>\overline{\mathcal{I}}_{\mathrm{s}_{1}}$ and $p_{\mathrm{s}_{1}} \geq p_{\mathrm{s}, \text { max }}$ is equivalent to $\overline{\mathcal{I}}_{\mathrm{s}} \leq \overline{\mathcal{I}}_{\mathrm{s}_{1}}$. On the other hand, $\mathcal{X} \leq 0 \Leftrightarrow P_{\mathrm{d}} \geq P_{\mathrm{d}_{1}}$, the constraint (41) is always valid, for every $p_{\mathrm{s}} \in\left[0, p_{\mathrm{s}, \mathrm{max}}\right]$. In this case, $P_{\mathrm{s}, \mathrm{opt}}=$ $p_{\mathrm{s}, \max }$.

\section{B. Improper Gaussian Signaling With Partial CSI Based De- sign}

In this section, we optimize the SU transmit power and circularity coefficient to maximize its achievable rate while maintaining an acceptable PU outage probability performance. Since we could not derive an exact value for the PU outage probability, we design our system considering an upper bound PU constraint. Therefore, we consider the following optimization problem,

$$
\begin{array}{cc}
\max _{p_{\mathrm{s}}, \mathcal{C}_{x}} & R_{\mathrm{s}}\left(p_{\mathrm{s}}, \mathcal{C}_{x}\right) \\
\text { subject to } & P_{\text {out }}^{\mathrm{UP}}\left(p_{\mathrm{s}}, \mathcal{C}_{x}\right) \leq P_{0} \\
& 0 \leq p_{\mathrm{s}} \leq p_{s, \max } \\
& 0 \leq \mathcal{C}_{x} \leq 1,
\end{array}
$$

where,

$$
P_{\text {out }}^{\mathrm{UP}}\left(p_{\mathrm{s}}, \mathcal{C}_{x}\right)=P_{\mathrm{d}} P_{1}+\left(1-P_{\mathrm{d}}\right) P_{2}^{\mathrm{UP}}\left(p_{\mathrm{s}}, \mathcal{C}_{x}\right) .
$$

Using the expression of the PU outage probability found in III-B and after some manipulations, the first constraint in (42) can be rewritten equivalently as follows,

$$
\exp \left(-\frac{\gamma_{p_{2}}\left(\overline{\mathcal{I}}_{\mathrm{s}}\right)}{\overline{\gamma_{\mathrm{p}}}}\right) \geq \mathcal{X}
$$

If $\mathcal{X} \leq 0 \Leftrightarrow P_{\mathrm{d}} \geq P_{\mathrm{d}_{1}}$, then, (44) is always valid for every $p_{\mathrm{s}} \in\left[0, p_{\mathrm{s}, \max }\right]$ and $\mathcal{C}_{x} \in[0,1]$. Therefore, beyond a certain detection performance, the SU can use a proper signal to maximize its achievable rate inferred by the optimal solution $\left(P_{\mathrm{s}, \text { opt }}, \mathcal{C}_{\mathrm{x}, \text { opt }}\right)=\left(p_{\mathrm{s}, \max }, 0\right)$. Otherwise, if $\mathcal{X}>0 \Leftrightarrow P_{\mathrm{d}}<$ $P_{\mathrm{d}_{1}}$, the constraint in (44) can be simplified as follows,

$$
h\left(p_{\mathrm{s}}, \mathcal{C}_{x}\right)=p_{\mathrm{s}}^{2} \overline{\mathcal{I}}_{\mathrm{s}}^{2} \varphi(0)\left(1-\mathcal{C}_{x}^{2}\right)+2 p_{\mathrm{s}} \overline{\mathcal{I}}_{\mathrm{s}} \varphi(1)+\varphi(2) \leq 0,
$$

where

$$
\varphi(x)=2^{2 R_{0}}-\left(1-\overline{\gamma_{\mathrm{p}}} p_{\mathrm{p}} \ln (\mathcal{X})\right)^{x} .
$$

As a first important result, we can state that if $\varphi(2) \geq$ $0 \Leftrightarrow \bar{\gamma}_{\mathrm{p}} \leq \bar{\gamma}_{\mathrm{p}_{1}}=-{\frac{\mu_{0}}{p_{\mathrm{p}} \ln (\mathcal{X})}}^{6}$, then $h\left(p_{\mathrm{s}}, \mathcal{C}_{x}\right)>0^{7}$ and the constraint will not be valid. The $\mathrm{SU}$, in this case, will remain silent. Therefore, and as expected, the operation of the SU is conditioned by the quality of the PU direct link. Otherwise,

\footnotetext{
${ }^{5} P_{\mathrm{d}_{1}}$ is insured to be between 0 and 1 by the feasibility condition stated in Theorem 3 .

${ }^{6} \bar{\gamma}_{\mathrm{p}_{1}}$ is proved to be always positive in Appendix $\mathrm{C}$

${ }^{7} \varphi(x)$ is decreasing in $x$, then, $\varphi(1)>0$
} 
the inequality in (45) can be solved and the SU can transmit its signal without causing an outage to the PU communication. We solve the following quadratic inequality,

$$
\Delta=4 \overline{\mathcal{I}}_{\mathrm{s}}^{2}\left(\varphi(1)^{2}-\left(1-\mathcal{C}_{x}^{2}\right) \varphi(0) \varphi(2)\right)>0 .
$$

The solution range is $p_{\mathrm{s}} \in\left[0, \tilde{p}_{\mathrm{s}}\left(\mathcal{C}_{x}\right)\right]$ where,

$$
\tilde{p}_{\mathrm{s}}\left(\mathcal{C}_{x}\right)=\frac{\sqrt{\varphi(1)^{2}-\left(1-\mathcal{C}_{x}^{2}\right) \varphi(0) \varphi(2)}-\varphi(1)}{\overline{\mathcal{I}}_{\mathrm{s}}\left(1-\mathcal{C}_{x}^{2}\right) \varphi(0)} .
$$

In this case, our optimization problem can be rewritten as follows,

$$
\begin{array}{cc}
\max _{p_{\mathrm{s}}, \mathcal{C}_{x}} & R_{\mathrm{s}}\left(p_{\mathrm{s}}, \mathcal{C}_{x}\right) \\
\text { subject to } & 0 \leq p_{\mathrm{s}} \leq \tilde{\mathcal{F}}\left(\mathcal{C}_{x}\right) \\
& 0 \leq \mathcal{C}_{x} \leq 1,
\end{array}
$$

where $\tilde{\mathcal{F}}\left(\mathcal{C}_{x}\right)=\min \left(p_{s, \text { max }}, \tilde{p}_{\mathrm{s}}\left(\mathcal{C}_{x}\right)\right)$. We opt for using the monotony of the objective function. Since, $R_{\mathrm{S}}\left(p_{\mathrm{s}}, \mathcal{C}_{x}\right)$ is an increasing function in $p_{\mathrm{s}}$, then $\tilde{\mathcal{F}}\left(\mathcal{C}_{x}\right)$ will be the best choice for $p_{\mathrm{s}}$, for all $\mathcal{C}_{x}$. Our design is simplified and we have an optimization problem depending on one parameter as follows,

$$
\begin{array}{cc}
\max _{\mathcal{C}_{x}} & R_{\mathrm{S}}\left(\tilde{\mathcal{F}}\left(\mathcal{C}_{x}\right), \mathcal{C}_{x}\right) \triangleq \tilde{R}_{\mathrm{s}}\left(\mathcal{C}_{x}\right) \\
\text { subject to } & 0 \leq \mathcal{C}_{x} \leq 1 .
\end{array}
$$

Since we obtained an optimization problem depending on one parameter, we can solve it according to the following theorem.

Theorem 4. In a feasible interweave cognitive radio system with IGS scheme adopted at the primary network and a PGS scheme at the secondary network operating at a target rate $R_{0}$ and a maximum outage probability $P_{0}$ for the primary communication, and assuming the availability of its link and the interference link from the $S U$ to the PU average CSI and the secondary links instantaneous CSI at the ST side, when the probability of detection is below a predefined threshold $P_{\mathrm{d}_{1}}$, the optimal SU transmit power and circularity coefficient will be set depending on the conditions stated in equation (50), where,

$$
\begin{aligned}
\overline{\mathcal{I}}_{\mathrm{s}_{2}} & =\max \left(\min \left(\frac{\varphi(1) \gamma_{\mathrm{s}}}{\varphi(0)}, \frac{\varphi(1) \gamma_{\mathrm{s}}}{\varphi(0)\left(1+p_{\mathrm{p}} I_{\mathrm{p}}\right)}\right), 0\right) \\
\overline{\mathcal{I}}_{\mathrm{s}_{3}} & =\max \left(\max \left(\frac{\varphi(1) \gamma_{\mathrm{s}}}{\varphi(0)}, \frac{\varphi(1) \gamma_{\mathrm{s}}}{\varphi(0)\left(1+p_{\mathrm{p}} I_{\mathrm{p}}\right)}\right), 0\right) \\
\mathcal{C}_{x}^{*} & =\sqrt{1+\frac{\varphi(2)+2 \varphi(1) p_{\mathrm{s}, \max } \overline{\mathcal{I}}_{\mathrm{s}}}{p_{\mathrm{s}, \max }^{2} \overline{\mathcal{I}}_{\mathrm{s}}^{2} \varphi(0)}} \\
\mathcal{C}_{x_{1}} & =\arg \max \left(R_{\mathrm{s}}(0), R_{\mathrm{s}}\left(\mathcal{C}_{x}^{* *}\right), \lim _{\mathcal{C}_{x} \rightarrow 1} R_{\mathrm{s}}\left(\mathcal{C}_{x}\right)\right) \\
\mathcal{C}_{x_{2}} & =\arg \max \left(R_{\mathrm{s}}(0), R_{\mathrm{s}}\left(\mathcal{C}_{x}^{* *}\right), R_{\mathrm{s}}\left(\mathcal{C}_{x}^{*}\right)\right) .
\end{aligned}
$$

Proof. Since $p_{\mathrm{s}}$ is increasing in $\mathcal{C}_{x}$, the value of $\tilde{\mathcal{F}}\left(\mathcal{C}_{x}\right)$ will depend on the position of $\tilde{p}_{\mathrm{s}}$ and $p_{\mathrm{s}, \max }$. We will use the fact that $R_{\mathrm{s}}$ is increasing in $p_{\mathrm{s}}$ and decreasing in $\mathcal{C}_{x}$ to choose the optimal pair. We can resume the results as follows:

Case 1: If $\tilde{p}_{\mathrm{s}}(0)>p_{\mathrm{s}, \max } \Leftrightarrow \overline{\mathcal{I}}_{\mathrm{s}}<\overline{\mathcal{I}}_{\mathrm{s}_{1}}$, then $\tilde{\mathcal{F}}\left(\mathcal{C}_{x}\right)=p_{\mathrm{s}, \max }$. Therefore, the optimal pair will be, $\left(p_{\mathrm{s}, \max }, 0\right)$.

Case 2: If $\lim _{\mathcal{C}_{x} \rightarrow 1} \tilde{p}_{\mathrm{s}}\left(\mathcal{C}_{x}\right)<p_{\mathrm{s}, \max }$. This limit is equal to $-\frac{\varphi(2)}{2 \overline{\mathcal{I}}_{\mathrm{s}} \varphi(1)}$ if $\varphi(1)>0$ and to positive infinity otherwise. Hence, $\tilde{\mathcal{F}}\left(\mathcal{C}_{x}\right)=\tilde{p}_{\mathrm{s}}\left(\mathcal{C}_{x}\right)$. In this case, we study the behavior of $\tilde{R}_{\mathrm{s}}\left(\mathcal{C}_{x}\right)$ which will have the following new expression,

$\tilde{R}_{\mathrm{s}}\left(\mathcal{C}_{x}\right)=A \log _{2}\left(a_{1} \tilde{p}_{\mathrm{s}}\left(\mathcal{C}_{x}\right)+a_{2}\right)+B \log _{2}\left(b_{1} \tilde{p}_{\mathrm{s}}\left(\mathcal{C}_{x}\right)+b_{2}\right)$,

${ }^{8}$ where, $a_{1}=2 \gamma_{\mathrm{s}}-\frac{2 \varphi(1) \gamma_{\mathrm{s}}^{2}}{\varphi(0) \mathcal{I}_{\mathrm{s}}}, a_{2}=1-\frac{\varphi(2) \gamma_{\mathrm{s}}^{2}}{\varphi(0) \mathcal{I}_{\mathrm{s}}^{2}}, b_{1}=\frac{2 \gamma_{\mathrm{s}}}{1+p_{\mathrm{p}} I_{\mathrm{p}}}-$ $\frac{2 \varphi(1) \gamma_{\mathrm{s}}^{2}}{\varphi(0) \overline{\mathcal{I}}_{\mathrm{s}}\left(1+p_{\mathrm{p}} I_{\mathrm{p}}\right)^{2}}$ and $b_{2}=1-\frac{\varphi(2) \gamma_{\mathrm{s}}^{2}}{\varphi(0) \overline{\mathcal{I}}_{\mathrm{s}}^{2}\left(1+p_{\mathrm{p}} I_{\mathrm{p}}\right)^{2}}$. The behavior of $\tilde{R}_{\mathrm{S}}$ will depend on the sign of $a_{1}$ and $b_{1}$ since $\tilde{p}_{\mathrm{S}}\left(\mathcal{C}_{x}\right)$ represents the only dependency of $\tilde{R}_{\mathrm{s}}$ on $\mathcal{C}_{x}$ and $\log _{2}(x)$ is an increasing function in $x>0$.

-If $a_{1}>0$ and $b_{1}>0 \Leftrightarrow \overline{\mathcal{I}}_{\mathrm{s}}>\overline{\mathcal{I}}_{1}=\frac{\varphi(1) \gamma_{\mathrm{s}}}{\varphi(0)}$ and $\overline{\mathcal{I}}_{\mathrm{s}}>\overline{\mathcal{I}}_{2}=$ $\frac{\varphi(1) \gamma_{\mathrm{s}}}{\varphi(0)\left(1+p_{\mathrm{p}} I_{\mathrm{p}}\right)}$ then $\tilde{R}_{\mathrm{S}}\left(\mathcal{C}_{x}\right)$ is increasing in $\mathcal{C}_{x}$ and the optimal solution is $\left(\lim _{\mathcal{C}_{x} \rightarrow 1} \tilde{p}_{\mathrm{s}}\left(\mathcal{C}_{x}\right), 1\right)$.

-If $a_{1} \leq 0$ and $b_{1} \leq 0 \Leftrightarrow \overline{\mathcal{I}}_{\mathrm{s}} \leq \overline{\mathcal{I}}_{1}$ and $\overline{\mathcal{I}}_{\mathrm{s}} \leq \overline{\mathcal{I}}_{2}$ then $\tilde{R}_{\mathrm{s}}\left(\mathcal{C}_{x}\right)$ is decreasing in $\mathcal{C}_{x}$ and the optimal solution is $\left(\tilde{p}_{\mathrm{s}}(0), 0\right)$.

-Else, we determine the stationary points of $\tilde{R}_{\mathrm{s}}\left(\mathcal{C}_{x}\right)$, compare their rates with those of the boundary points and then pick up the pair $\left(\tilde{p}_{\mathrm{s}}, \mathcal{C}_{x}\right)$ that maximizes $\tilde{R}_{\mathrm{s}}$. $\tilde{R}_{\mathrm{S}}$ has one stationary point characterized by,

$$
p_{\mathrm{ss}}=-\frac{a_{1} b_{2} A+b_{1} a_{2} B}{a_{1} b_{1}(A+B)} .
$$

If $\left.p_{\mathrm{ss}} \in\right] \tilde{p}_{\mathrm{s}}(0), \lim _{\mathcal{C}_{x} \rightarrow 1} \tilde{p}_{\mathrm{s}}\left(\mathcal{C}_{x}\right)\left[, \quad\right.$ then, $\mathcal{C}_{x}^{* *}=$

Case 3: $\tilde{p}_{\mathrm{s}}(0) \leq p_{\mathrm{s}, \max } \leq \lim _{\mathcal{C}_{x} \rightarrow 1} \tilde{p}_{\mathrm{s}}\left(\mathcal{C}_{x}\right) \Rightarrow$ we have one intersection point $\mathcal{C}_{x}^{*}=\sqrt{1-\frac{\varphi(2)+2 \varphi(1) p_{\mathrm{s}, \max } I_{\mathrm{s}}}{-p_{\mathrm{s}, \max }^{2} I_{\mathrm{s}}^{2} \varphi(0)}}$

In this case, the expression of the achievable rate will be,

$$
\tilde{R}_{\mathrm{s}}\left(\mathcal{C}_{x}\right)= \begin{cases}R_{\mathrm{s}}\left(\tilde{p}_{\mathrm{s}}\left(\mathcal{C}_{x}\right), \mathcal{C}_{x}\right) & 0 \leq \mathcal{C}_{x} \leq \mathcal{C}_{x}^{*} \\ R_{\mathrm{s}}\left(p_{\mathrm{s}, \max }, \mathcal{C}_{x}\right) & \mathcal{C}_{x}^{*}<\mathcal{C}_{x} \leq 1\end{cases}
$$

For $\mathcal{C}_{x} \in\left[\mathcal{C}_{x}^{*}, 1\right] \max \tilde{R}_{\mathrm{s}}=\tilde{R}_{\mathrm{s}}\left(\mathcal{C}_{x}^{*}\right)$ since $\tilde{p}_{\mathrm{s}}$ is constant and $\tilde{R}_{\mathrm{S}}$ is decreasing in $\mathcal{C}_{x}$. Then,

$$
\max _{\mathcal{C}_{x} \in[0,1]} \tilde{R}_{\mathrm{s}}\left(\mathcal{C}_{x}\right)=\max _{\mathcal{C}_{x} \in\left[0, \mathcal{C}_{x}^{*}\right]} R_{\mathrm{s}}\left(\tilde{p}_{\mathrm{s}}\left(\mathcal{C}_{x}\right), \mathcal{C}_{x}\right) .
$$

${ }^{8} \mathrm{We}$ follow the same reasoning as in section IV-B to find this expression.

$$
\left(P_{\mathrm{s}, \text { opt }}, \mathcal{C}_{\mathrm{x}, \mathrm{opt}}\right)= \begin{cases}\left(p_{\mathrm{s}, \max }, 0\right) & \tilde{p}_{\mathrm{s}}(0)>p_{\mathrm{s}, \max } \\ \left(\tilde{p}_{\mathrm{s}}(0), 0\right) & \tilde{p}_{\mathrm{s}}(0)<p_{\mathrm{s}, \max } \text { and } \overline{\mathcal{I}}_{\mathrm{s}}<\overline{\mathcal{I}}_{\mathrm{s}_{1}} \overline{\mathcal{I}}_{\mathrm{s}}>\overline{\mathcal{I}}_{\mathrm{s}_{2}} \\ \left(\lim _{\mathcal{C}_{x} \rightarrow 1} \tilde{p}_{\mathrm{s}}\left(\mathcal{C}_{x}\right), 1\right) & \lim _{\mathcal{C}_{x} \rightarrow 1} \hat{p}_{\mathrm{s}}\left(\mathcal{C}_{x}\right)<p_{\mathrm{s}, \max } \text { and } \\ \left(\tilde{p}_{\mathrm{s}}\left(\mathcal{C}_{x_{1}}\right), \mathcal{C}_{x_{1}}\right) & \lim _{\mathcal{C}_{x} \rightarrow 1} \tilde{p}_{\mathrm{s}}\left(\mathcal{C}_{x}\right)<p_{\mathrm{s}, \max } \text { and } \overline{\mathcal{I}}_{\mathrm{s}_{1} \leq \overline{\mathcal{I}}_{\mathrm{s}} \leq \overline{\mathcal{I}}_{\mathrm{s}_{2}}} \\ \left(p_{\mathrm{s}, \max }, \mathcal{C}_{x}^{*}\right) & \tilde{p}_{\mathrm{s}}(0)<p_{\mathrm{s}, \max }<\lim _{\mathcal{C}_{x} \rightarrow 1} \tilde{p}_{\mathrm{s}}\left(\mathcal{C}_{x}\right) \text { and } \overline{\mathcal{I}}_{\mathrm{s}}>\overline{\mathcal{I}}_{\mathrm{s}_{2}} \\ \left(\tilde{p}_{\mathrm{s}}\left(\mathcal{C}_{x_{2}}\right), \mathcal{C}_{x_{2}}\right) & \tilde{p}_{\mathrm{s}}(0)<p_{\mathrm{s}, \max }<\lim _{\mathcal{C}_{x} \rightarrow 1} \tilde{p}_{\mathrm{s}}\left(\mathcal{C}_{x}\right) \text { and } \overline{\mathcal{I}}_{\mathrm{s}_{1} \leq \overline{\mathcal{I}}_{\mathrm{s}} \leq \overline{\mathcal{I}}_{\mathrm{s}_{2}}}\end{cases}
$$




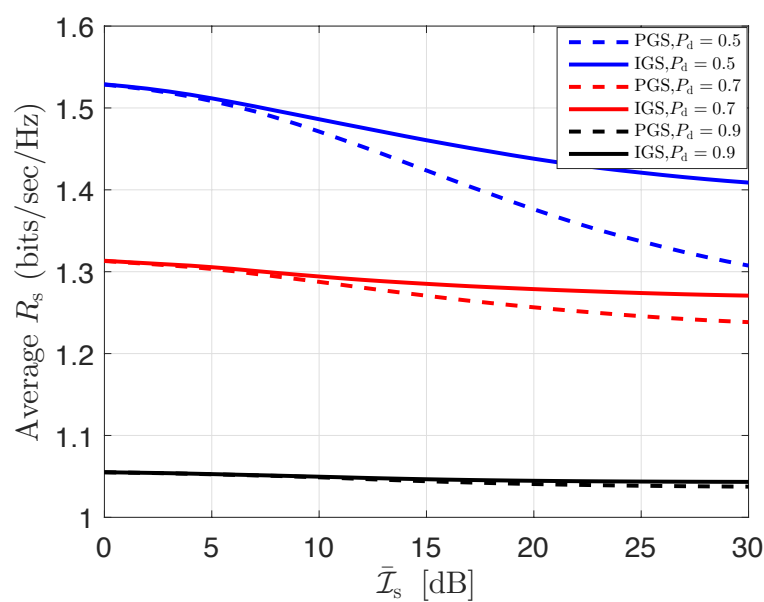

(a) $p=0.3$

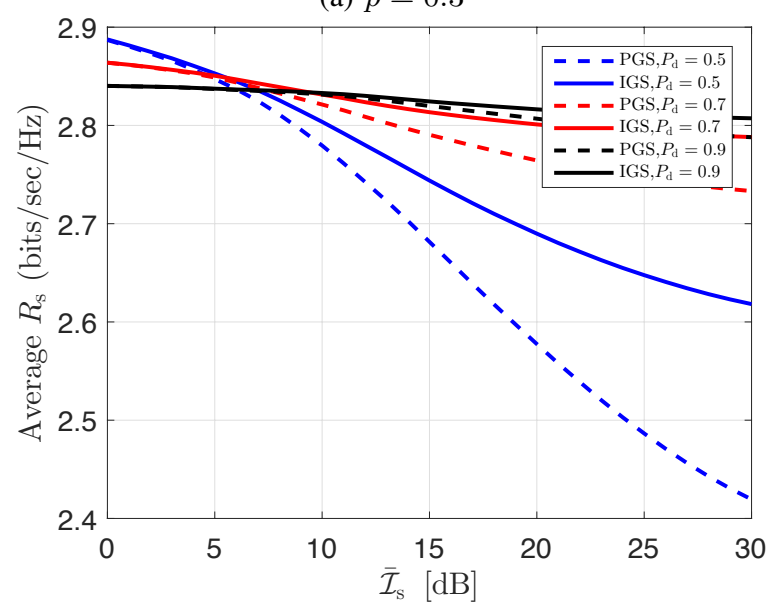

(b) $p=0.9$

Figure 2: $R_{\mathrm{S}}$ versus $\overline{\mathcal{I}}_{\mathrm{s}}$ for both PGS and IGS based schemes for different values of $P_{\mathrm{d}}$ and for different $p$.

This case will be resolved as Case 2 after considering $\left[0, \mathcal{C}_{x}^{*}\right]$ as a range for $\mathcal{C}_{x}$.

These results support the known conditions for improper signal operation which is its suitability for systems with high interference links from the SU to the PU. We can easily deduce that the system opts for a proper solution for low values of $\overline{\mathcal{I}}_{\mathrm{s}}$ and to the maximum possible impropriety degree in higher values of interference. Furthermore, for low direct PU link performance, the SU increases its circularity coefficient to increase its transmit power and reduce its effect on the primary communication.

\section{Opportunistic System Design}

In a full CSI based design, the IGS captures the PGS design, so if IGS does not give benefit, the solution will reduce to PGS. However, the scenario is not the same in partial-CSI based design because it is based on upper bound on the outage probability. Thus, IGS based design may give a solution that is worse than PGS. Therefore, under some conditions, the PGS outperforms the IGS leading to the need for an opportunistic design that intelligently chooses the scheme (proper or improper) in order to maximize the SU achievable rate. This design is proposed to deal with the side-effect of upper bound.

\section{Design challenges and future work}

The designed system, like all cognitive radio systems, has to deal with some technical challenges, in addition to, challenges related to using suitable sensing mechanism, including detection algorithms. Unlike underlay CR that does not require detection, it is considered a decisive and major phase in this interweave CR. Moreover, for more complex scenarios, such as a multiuser cognitive radio network, the decision making, whether central or distributed, affects the performance. Another critical challenge is to ensure the secure operation of all the existing agents, though less severe in interweave networks than in overlay setup. As a progression of this work, one can move forward and investigate the case of several licensed and unlicensed Multiple-Input Multiple-Output (MIMO) users. However, dealing with multiuser network in interweave CR will invoke new design parameters, mainly the decision making and the different activity recurrence of primary users.

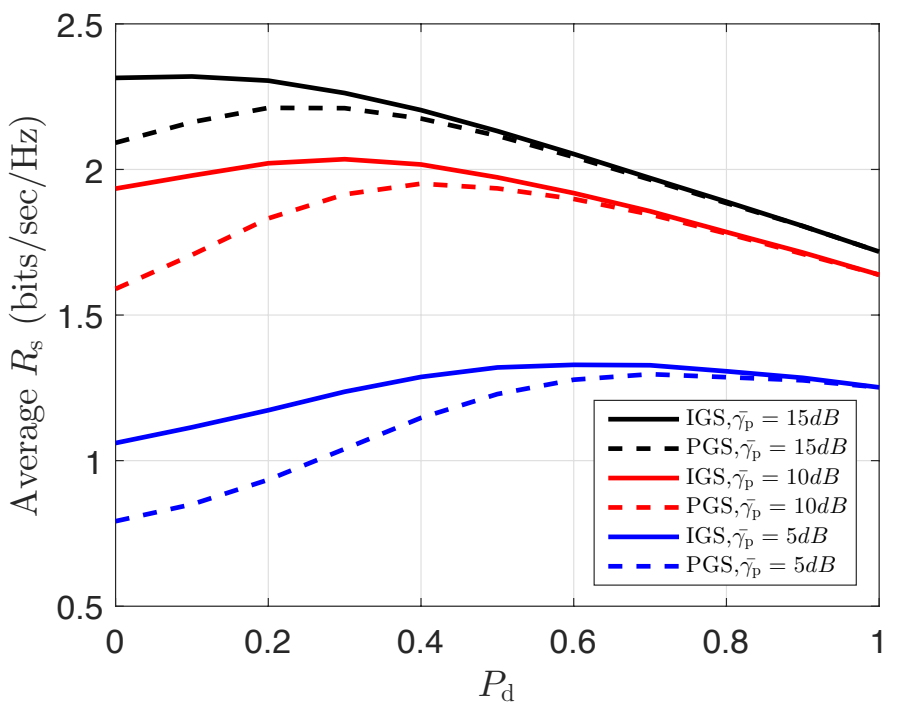

Figure 3: $R_{\mathrm{S}}$ versus $P_{\mathrm{d}}$ for both PGS and IGS based schemes for different values of $\bar{\gamma}_{\mathrm{p}}$.

\section{Simulation Results}

In this section, we present different simulation results to evaluate the gain that is reaped from employing IGS scheme as a function of the system's parameters for perfect and partial CSI. We run Monte-Carlo simulation and take the average over different CSI values. In the following, we consider $p_{\mathrm{p}}=0 \mathrm{~dB}$, $p_{\mathrm{s}, \max }=0 \mathrm{~dB}, P_{\mathrm{fa}}=0.1$ and $\frac{T-\tau}{T}=0.9$.

\section{A. Results for Perfect CSI Based Design}

As for the simulation parameters, we use the following simulation parameters, unless otherwise specified; $\overline{\gamma_{\mathrm{p}}}=10 \mathrm{~dB}$, $\overline{\mathcal{I}}_{\mathrm{p}}=10 \mathrm{~dB}, \bar{\gamma}_{\mathrm{s}}=15 \mathrm{~dB}, \overline{\mathcal{I}}_{\mathrm{s}}=15 \mathrm{~dB}$ and $R_{\min }=1 \mathrm{~b} / \mathrm{s} / \mathrm{Hz}$. Both $P_{\mathrm{d}}$ and $p$ will be specified in each example. 


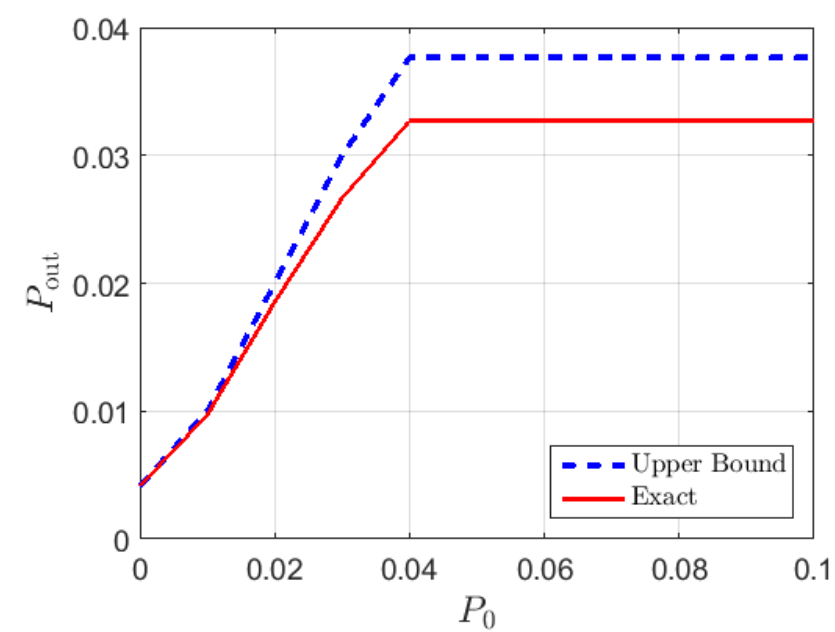

Figure 4: Comparison between the exact PUs outage probability and the upper bound versus $P_{0}$.

Example 1: In the first example, assuming various $\mathrm{PU}$ activities, we examine the SU achievable rate performance as a function of the SU interference on the PU communication. For this purpose, we plot the average $R_{\mathrm{S}}$ versus $\overline{\mathcal{I}}_{\mathrm{s}}$ for different values of $P_{\mathrm{d}}$ in Fig.2a and Fig.2b. First, we assume the PU is often active with a probability 0.7 to access the spectrum in Fig.2a. We observe a degradation of the average $R_{\mathrm{s}}$ as the interference $\overline{\mathcal{I}}_{\mathrm{s}}$ increases as a consequence of diminishing the SU opportunity to access the spectrum with strong interference levels on the PU. According to the PU QoS requirement, the SU achieves almost constant requirement when it has high sensing and detection capabilities, as a result of the relatively substantial PU achievable rate compared to $R_{\text {min }}$. Thus, the PU performance will not be deteriorated if the SU accesses the spectrum with wrong detection. This behavior is proved for lower SU sensing system. Since the PU QoS requirement is achieved, the SU can access the spectrum and achieve a higher rate even if the PU is active. Thus, if the SU accesses the spectrum when the PU is active, it will not deteriorate the PU performance. This behavior is confirmed for lower SU sensing system, where the SU can access the spectrum even if it is occupied achieving higher rate since the PU rate requirement is met. Under the same lower sensing quality assumption, the $\mathrm{SU}$ is enforced to reduce its transmit power and reach a lower rate because of the increase of harmful interference to the PU. Therefore, the SU rate is reduced with the increase of $\overline{\mathcal{I}}_{\mathrm{S}}$.

As for the relative performance between PGS and IGS, the same performance is achieved with excellent sensing system since the system becomes interference-free and the PGS is optimal in this case. On the other hand, at low detection capabilities, the interference channels get stronger and the IGS emerges as an optimal scheme. Fig. 2b shows the same results but for PU activity of 0.1 . In this case, the average $R_{\mathrm{S}}$ decreases with the interference regardless of the probability of detection. Interestingly, the gap between different SU detecting quality is small due to the low PU activity, at low values of $\overline{\mathcal{I}}_{\mathrm{s}}$. Example 2: In this example, we study the joint effect of the accuracy of the detection system and the PU channel quality.
To this end, we plot the average $R_{\mathrm{s}}$ versus $P_{\mathrm{d}}$ for different $\bar{\gamma}_{\mathrm{p}}$ and PU activity with $p=0.5$ in Fig.3. First, we observe that as the PU direct link strength increases, it allows a room for the SU to send its message even for lower sensing capabilities. Here, the PU QoS is not affected by the introduced interference. As for the relative performance between the IGS and PGS based schemes, the merit of employing IGS appears at low $P_{\mathrm{d}}$ since the interference increases with lower SU sensing capabilities. In this case, the system can adopt IGS scheme to compensate for the interference impact on the PU, which boosts $R_{\mathrm{s}}$ over the PGS scheme. Furthermore, the benefit of IGS is significant and the performance is improved when $P_{\mathrm{d}}$ and $\bar{\gamma}_{\mathrm{p}}$ are low, causing a severe interference impact on the PU activity. In fact, when $\bar{\gamma}_{\mathrm{p}}$ has a low gain, the IGS scheme can improve the SU rate and compensate for its interference by increasing its circularity coefficient.

\section{B. Results for Partial CSI Based Design}

As for the simulation parameters, we use the following simulation parameters, unless otherwise specified; $p_{\mathrm{p}}=0 \mathrm{~dB}$, $p_{\mathrm{s}, \max }=0 \mathrm{~dB}, \bar{\gamma}_{\mathrm{p}}=20 \mathrm{~dB}, \overline{\mathcal{I}}_{\mathrm{p}}=10 \mathrm{~dB}, \bar{\gamma}_{\mathrm{s}}=15 \mathrm{~dB}$, $\overline{\mathcal{I}}_{\mathrm{s}}=20 \mathrm{~dB}$ and $R_{\text {min }}=0.5 \mathrm{~b} / \mathrm{s} / \mathrm{Hz}$. Concerning the characteristic parameters of the interweave, we consider $P_{\mathrm{fa}}=0.1$ and $\frac{T-\tau}{T}=0.9, P_{\mathrm{d}}=0.9$ since the detection probability is a critical parameter to the PU communication and $p=0.5$.

Example 3: In this example, we compare the upper bound found in (43) and (25) with the exact value computed numerically from (23) and (19) and assuming the optimal values of $p_{\mathrm{s}}$ and $\mathcal{C}_{x}$ found from problem (42). We plot $P_{\text {out,p }}$ versus $P_{0}$ in Fig. 4 and we found that the upper bound is very tight to the exact outage probability for small $P_{0}$ values.

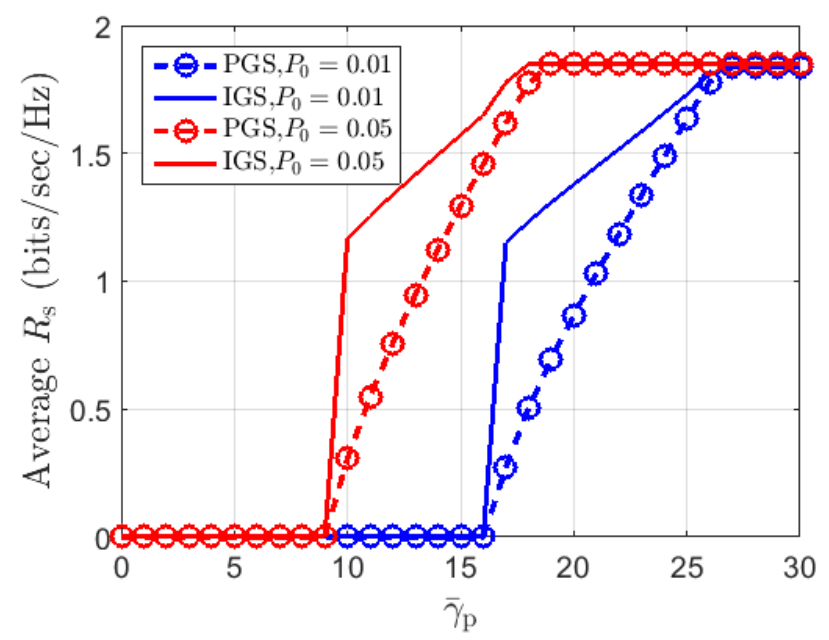

Figure 5: $R_{\mathrm{s}}$ versus $\bar{\gamma}_{\mathrm{p}}$ for both PGS and IGS based schemes for different values of $P_{0}$.

Example 4: In this example, we study the SU achievable rate versus the PU direct link for different maximum outage probability values. To this end, we plot the $R_{\mathrm{s}}$ versus $\bar{\gamma}_{\mathrm{p}}$ for $P_{0}=0.01$ and $P_{0}=0.05$ in Fig. 5. First, we observe that $R_{\mathrm{s}}$ increases with the increase of the PU direct link CNR, giving the ST a bigger room to transmit its signal without causing an 


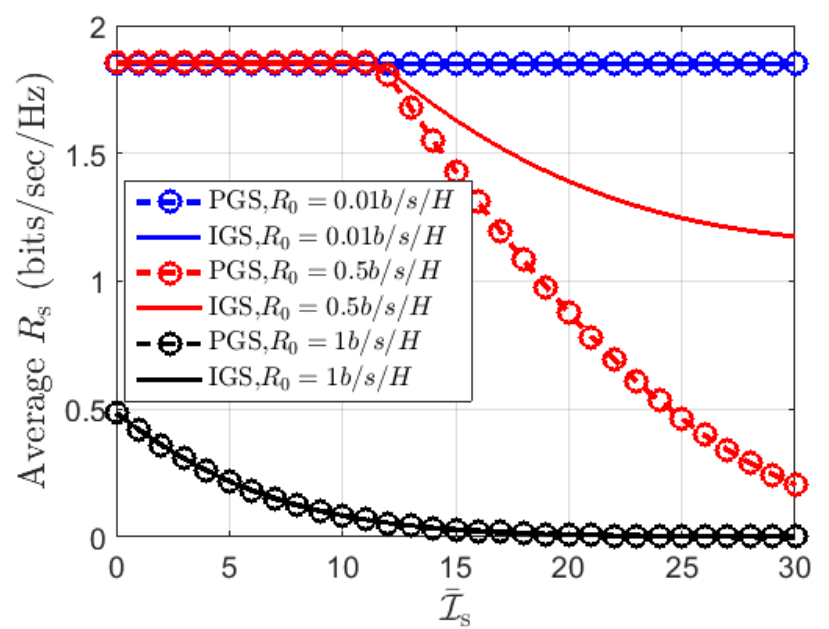

Figure 6: $R_{\mathrm{S}}$ versus $\overline{\mathcal{I}}_{\mathrm{S}}$ for both PGS and IGS based schemes for different values of $R_{0}$.

outage to the PU. On the other hand, for $P_{0}=0.01$, the SU remains silent for higher $\bar{\gamma}_{\mathrm{p}}$ than for $P_{0}=0.05$. Therefore, higher requirements for the PU communication reduces the possibilities for the SU to transmit its signal. As specified in the previous section, for lower values of $\bar{\gamma}_{\mathrm{p}}$, in the PGS scheme, the SU is forced to decrease its transmit power to meet the PU QoS requirements, while in the IGS scheme, the SU can increase its transmit power and reduce its interference by increasing its circularity coefficient.

Example 5: In this example, we explore the impact of the interference on the SU performance. We plot in Fig. 6, the SU achievable rate versus $\overline{\mathcal{I}}_{\mathrm{s}}$ for different values of minimum PU achievable rate $R_{0}$. We can infer that for low values of $R_{0}$, the $\mathrm{SU}$ can operate freely and achieve higher rates. The IGS scheme reduces to the PGS one in this case, since the ST can use its maximum power while meeting the PU QoS requirements. For $R_{0}=0.5 \mathrm{~b} / \mathrm{s} / \mathrm{H}$, the IGS outperforms considerably the PGS, for high interference values from the SU. In this case, the PGS decreases its transmit power while the IGS can increase the SU transmit power and compensate its effect by increasing its circularity coefficient. For higher $R_{0}$, the SU will remain silent most of the time especially for high $\overline{\mathcal{I}}_{\mathrm{s}}$ to guarantee a reliable communication for the PU.

\section{Comparison between Perfect-CSI and Partial-CSI based designs}

Example 6: In this example, we plot the $\mathrm{SU}$ achievable rate versus its direct link for different values of $\bar{\gamma}_{\mathrm{p}}$ in Fig.8. Unlike the case of perfect CSI system, for low PU direct link capabilities, the SU remains silent to meet the PU requirements and because it is ignorant of the instantaneous state of the PU link and how much the SU signal can harm the PU communication. For $\bar{\gamma}_{\mathrm{p}}=20 \mathrm{~dB}$, the gap between the PGS and IGS scheme becomes significant since the improper signaling design can increase the SU transmit power and compensate for its effect by increasing its circularity coefficient, while the proper design decreases its transmit power to maintain the

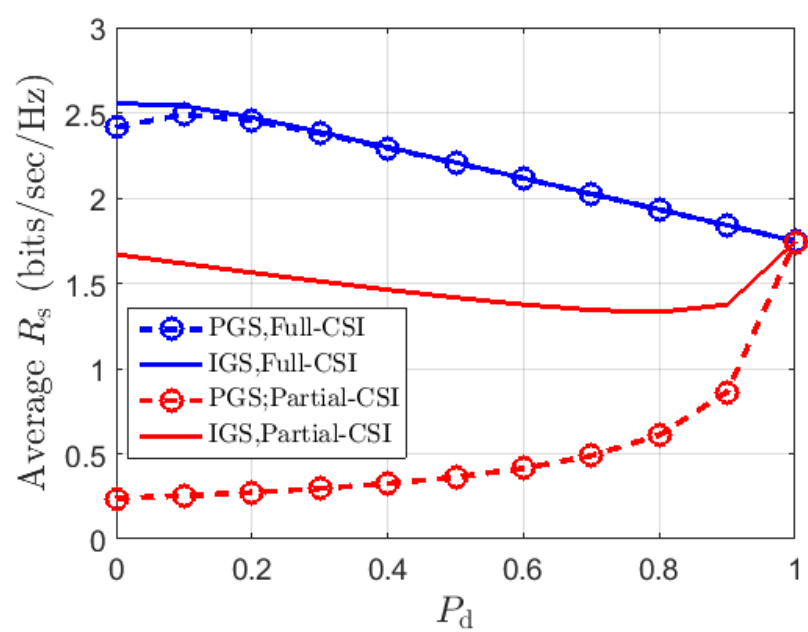

Figure 7: $R_{\mathrm{S}}$ versus $P_{\mathrm{d}}$ for both PGS and IGS based schemes for Full-CSI and Partial-CSI.

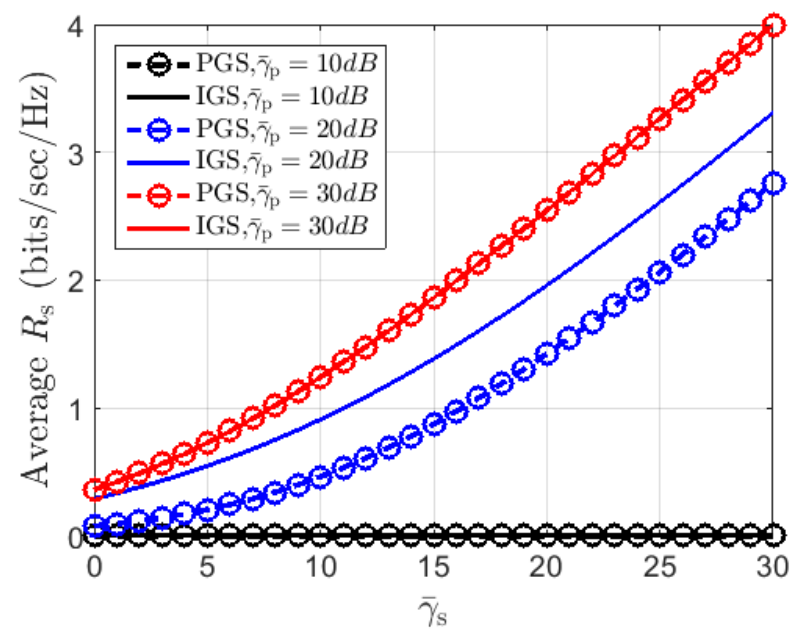

Figure 8: $R_{\mathrm{S}}$ versus $\bar{\gamma}_{\mathrm{s}}$ for both PGS and IGS based schemes for different values of $\bar{\gamma}_{p}$.

minimum required performance for the PU. For $\bar{\gamma}_{\mathrm{p}}=30 \mathrm{~dB}$, both proper and improper schemes reduce to the same solution, the SU can use its maximum power budget while meeting the PU QoS requirements due to the good quality of its direct link.

Example 7: In this example, we investigate the interweave system performance while changing the detection capabilities. We plot in Fig. $7 R_{\mathrm{s}}$ versus $P_{\mathrm{d}}$ for both Full and Partial CSI designs. First, it is worth to state that the Partial-CSI system cannot achieve the performance of the Full-CSI system since the uncertainty on the unknown links gives the SU a smaller room to transmit its signal and thus achieve lower rates. Assuming a good PU direct link, the IGS scheme in the Full-CSI system reduces to the PGS one, as derived from the first section. On the other hand, the IGS scheme in the PartialCSI based system achieves a noticeable performance than the PGS even for high detection abilities. In fact, the uncertainty on the unknown links forces the PGS scheme to decrease its 
transmit power to meet the PU QoS requirements while in the IGS scheme, the SU can increase its transmit power and compensate its effect by increasing its circularity coefficient.

Example 8: Since the interference from the SU to the $\mathrm{PU}$ is an important parameter in designing our system, we investigate the differences between the PGS and IGS schemes. As such, we plot $R_{\mathrm{s}}$ versus $\overline{\mathcal{I}}_{\mathrm{s}}$ for both the full and Partial CSI based designs in Fig. 9. We can observe that PGS and IGS schemes are equivalent in the instantaneous CSI system while the benefit reaped from employing IGS can be easily recognized in the partial CSI system. As clarified above, the $\mathrm{SU}$, in the improper signaling based design, can increase its transmit power and compensate its effect by increasing its circularity coefficient. Besides, employing an upper bound for the PU outage probability reduces the feasible space of the optimization problem and therefore, the optimized achievable rate decreases.

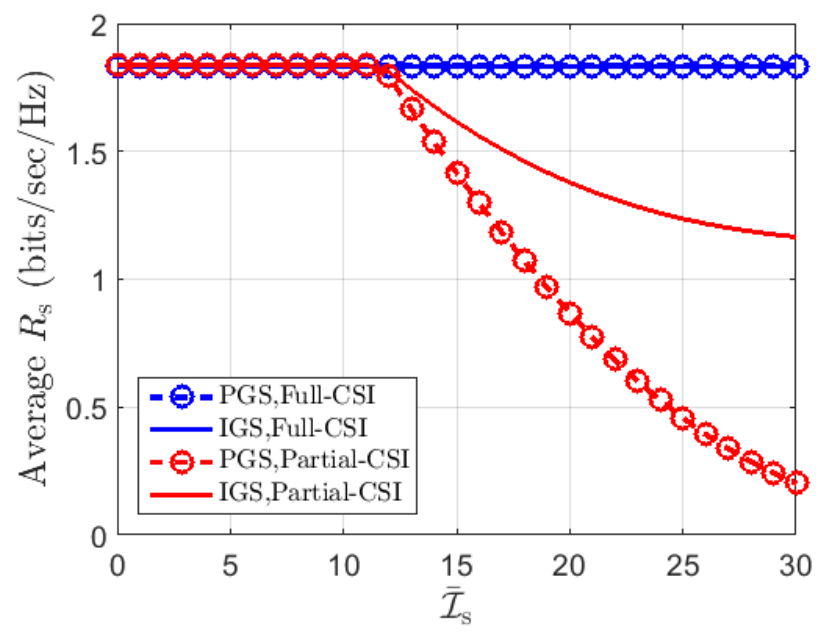

Figure 9: $R_{\mathrm{s}}$ versus $\overline{\mathcal{I}}_{\mathrm{s}}$ for both PGS and IGS based schemes for Full-CSI and Partial-CSI.

\section{CONCLUSION}

In this paper, we have considered an interweave cognitive radio system with IGS employed at the SU side and PGS at the PU side. We have expressed the achievable rate of both the PU and the SU based on a predefined detection and false alarm probabilities. Then, we proposed an optimal solution for the power allocation and the circularity coefficient that maximizes the SU achievable rate while satisfying a minimum QoS requirement for the PU. In the beginning, we considered an instantaneous CSI of all links in the ST side. Then, we assumed a more general and practical case of partial CSI knowledge. We have derived the optimal solution for PGS scheme at the SU to be used as a benchmark for the IGS system performance. We have introduced different simulation examples that showed the main advantage of the IGS scheme in several scenarios including low detection probabilities, high interference link to the PU and weak PU direct channels..

\section{APPENDIX A \\ CONVEXITY PROOF OF $\omega(x)$}

To decide on the convexity of $\omega$, we compute its second derivative. $\omega$ is in the form of $\exp (\beta)$ where $\beta(x)=\frac{-2^{R_{0}} \sqrt{a\left(\mathcal{C}_{x}\right) p_{\mathrm{s}}^{2} x^{2}+2 p_{\mathrm{s}} x+1}+p_{\mathrm{s}} x+1}{p_{\mathrm{p}} \gamma_{\mathrm{p}}}$. Then, $\frac{\partial^{2} \omega}{\partial x^{2}}=$ $\exp (\beta(x))\left(\frac{\partial^{2} \beta}{\partial x^{2}}+\left(\frac{\partial \beta}{\partial x}\right)^{2}\right)$. We have $\left(\frac{\partial \beta}{\partial x}\right)^{2}>0$ and $\frac{\partial^{2} \beta}{\partial x^{2}}=$ $\frac{2 R_{0} / p_{\mathrm{p}} \gamma_{\mathrm{p}}}{\left(a\left(\mathcal{C}_{x}\right) p_{\mathrm{s}}^{2} x^{2}+2 p_{\mathrm{s}} x+1\right)^{\frac{3}{2}}}\left(1-a\left(\mathcal{C}_{x}\right)\right)>0$ since $1-a\left(\mathcal{C}_{x}\right)=$ $\left(1-\frac{1}{2^{2 R_{0}}}\right) \mathcal{C}_{x}^{2} \geq 0$. Therefore $\frac{\partial^{2} \omega}{\partial x^{2}}>0$.

\section{APPENDIX B \\ EQUIVALENT EXPRESSION PROOF OF $R_{\mathrm{S}}$}

The transmit power expression found in (32) satisfies the equality in (30), then we can state that, $\hat{p}_{\mathrm{s}}^{2}=-\frac{2 \hat{p}_{\mathrm{s}} \mathcal{I}_{\mathrm{s}} \psi(1)+\psi(2)}{\mathcal{I}_{\mathrm{s}}^{2}\left(1-\mathcal{C}_{x}^{2}\right) \psi(0)}$. By replacing $\hat{p}_{\mathrm{s}}^{2}$ in the rate expression (5), we find a simplified expression (36).

\section{APPENDIX C \\ Strictly Positive Characteristics Proof of $\bar{\gamma}_{\mathrm{p}_{1}}$}

We have $\bar{\gamma}_{\mathrm{p}_{1}}>0$ if and only if $\log (\mathcal{X})<0$, i.e. $\mathcal{X}<1$ which is equivalent to $1-\mathcal{X}>0$. Since $1-\mathcal{X}=\frac{P_{0}-P_{\mathrm{d}} P_{1}}{1-P_{\mathrm{d}}}$ then $1-\mathcal{X}>0$ if and only if $P_{\mathrm{d}}<\frac{P_{0}}{P_{1}} \cdot \frac{P_{0}}{P_{1}}>1$ because $P_{1}<P_{0}$ Therefore, $1-\mathcal{X}$ is always positive.

\section{REFERENCES}

[1] Y.-C. Liang, K.-C. Chen, G. Y. Li, and P. Mähönen, "Cognitive radio networking and communications: An overview," IEEE Trans. Veh. Tech., vol. 60, no. 7, pp. 3386-3407, Sept. 2011.

[2] A. Goldsmith, S. A. Jafar, I. Maric, and S. Srinivasa, "Breaking spectrum gridlock with cognitive radios: An information theoretic perspective," Proc. IEEE, vol. 97, no. 5, pp. 894-914, May. 2009.

[3] F. F. Digham, M.-S. Alouini, and M. K. Simon, "On the energy detection of unknown signals over fading channels," IEEE Trans. Commun., vol. 55, no. 1, pp. 21-24, Jan. 2007.

[4] H. Urkowitz, "Energy detection of unknown deterministic signals," Proc. IEEE, vol. 55, no. 4, pp. 523-531, Apr. 1967.

[5] A. Mariani, A. Giorgetti, and M. Chiani, "Energy detector design for cognitive radio applications," in Proc. IEEE Int. Waveform Diversity and Design Conf. (WDD). IEEE, Aug. 2010.

[6] F. Salahdine, H. El Ghazi, N. Kaabouch, and W. F. Fihri, "Matched filter detection with dynamic threshold for cognitive radio networks," in Proc. Int. Wireless Networks and Mobile Commun. (WINCOM). IEEE, Oct. 2015.

[7] S. Shobana, R. Saravanan, and R. Muthaiah, "Matched filter based spectrum sensing on cognitive radio for ofdm wlans," Int. Journal of Engineering and Tech., vol. 5, no. 1, pp. 142-146, Feb. 2013.

[8] A. Kaushik, S. K. Sharma, S. Chatzinotas, B. Ottersten, and F. K. Jondral, "Sensing-throughput tradeoff for interweave cognitive radio system: A deployment-centric viewpoint," IEEE Trans. Wireless Commun., vol. 15, no. 5, pp. 3690-3702, May. 2016.

[9] M. C. Filippou, D. Gesbert, and G. A. Ropokis, "A comparative performance analysis of interweave and underlay multi-antenna cognitive radio networks," IEEE Trans. Wireless Commun., vol. 14, no. 5, pp. 2911-2925, May. 2015.

[10] A. Kariminezhad, A. Sezgin, and M. Pesavento, "Power efficiency of improper signaling in mimo full-duplex relaying for k-user interference networks," in Proc. IEEE Int. Conf. Commun. (ICC). IEEE, May. 2017, pp. 1-6.

[11] F. D. Neeser and J. L. Massey, "Proper complex random processes with applications to information theory," IEEE Trans. Inf. Theory, vol. 39, no. 4, pp. 1293-1302, Jul. 1993.

[12] S. Javed, O. Amin, B. Shihada, and M.-S. Alouini, "A Journey from improper Gaussian signaling to asymmetric signaling," 2019. [Online]. Available: http://hdl.handle.net/10754/656037 
[13] P. J. Schreier and L. L. Scharf, Statistical signal processing of complexvalued data: the theory of improper and noncircular signals. Cambridge University Press, 2010.

[14] Z. K. Ho and E. Jorswieck, "Improper gaussian signaling on the twouser siso interference channel," IEEE Trans. Wireless Commun., vol. 11, no. 9, pp. 3194-3203, Sept. 2012.

[15] Y. Zeng, C. M. Yetis, E. Gunawan, Y. L. Guan, and R. Zhang, "Transmit optimization with improper gaussian signaling for interference channels," IEEE Trans. Signal Process., vol. 61, no. 11, pp. 2899-2913, Jun. 2013

[16] Y. Zeng, R. Zhang, E. Gunawan, and Y. L. Guan, "Optimized transmission with improper gaussian signaling in the k-user miso interference channel," IEEE Trans. Wireless Commun., vol. 12, no. 12, pp. 6303 6313, Dec. 2013.

[17] E. Kurniawan and S. Sun, "Improper gaussian signaling scheme for the z-interference channel," IEEE Trans. Wireless Commun., vol. 14, no. 7, pp. 3912-3923, Jul. 2015

[18] S. Lagen, A. Agustin, and J. Vidal, "On the superiority of improper gaussian signaling in wireless interference mimo scenarios," IEEE Trans. Commun., vol. 64, no. 8, pp. 3350-3368, Aug. 2016.

[19] C. Lameiro, I. Santamaría, and P. J. Schreier, "Rate region boundary of the siso z-interference channel with improper signaling," IEEE Trans. Commun., vol. 65, no. 3, pp. 1022-1034, Mar. 2017.

[20] C. Lameiro and I. Santamaría, "Degrees-of-freedom for the 4-user siso interference channel with improper signaling," in IEEE Int. Conf. Commun. (ICC). IEEE, Jun. 2013, pp. 3053-3057.

[21] S. A. Jafar and S. Shamai, "Degrees of freedom region of the mimo $x$ channel," IEEE Trans. Inf. Theory, vol. 54, no. 1, pp. 151-170, Jan. 2008.

[22] I. A. Mahady, E. Bedeer, S. Ikki, and H. Yanikomeroglu, "Sum-rate maximization of noma systems under imperfect successive interference cancellation," IEEE Commun. Lett., vol. 23, no. 3, pp. 474-477, Mar. 2019.

[23] M. Gaafar, M. G. Khafagy, O. Amin, and M.-S. Alouini, "Improper gaussian signaling in full-duplex relay channels with residual selfinterference," in Proc. IEEE Int. Conf. Commun. (ICC). IEEE, May. 2016, pp. 1-7.

[24] S. Javed, O. Amin, S. S. Ikki, and M.-S. Alouini, "Impact of improper gaussian signaling on hardware impaired systems," in Proc. IEEE Int. Conf. Commun. (ICC). IEEE, May. 2017, pp. 1-6.

[25] _ "On the optimal detection and error performance analysis of the hardware impaired systems," in Proc. IEEE Global Commun. Conf. (GLOBECOM). IEEE, Dec. 2017, pp. 1-7.

[26] — "Asymmetric modulation for hardware impaired systems-error probability analysis and receiver design," IEEE Trans. Wireless Commun., vol. 18, no. 3, pp. 1723-1738, Mar. 2019.

[27] S. Javed, O. Amin, B. Shihada, and M.-S. Alouini, "Improper gaussian signaling for hardware impaired multihop full-duplex relaying systems," IEEE Trans. Commun., vol. 67, no. 3, pp. 1858-1871, Mar. 2018.

[28] S. Javed, O. Amin, S. S. Ikki, and M.-S. Alouini, "Multiple antenna systems with hardware impairments: New performance limits," IEEE Trans. Veh. Tech., vol. 68, no. 2, pp. 1593-1606, Feb. 2019.

[29] C. Lameiro, I. Santamaría, and P. J. Schreier, "Benefits of improper signaling for underlay cognitive radio," IEEE Wireless Commun. Lett., vol. 4, no. 1, pp. 22-25, Feb. 2015.

[30] _ , "Analysis of maximally improper signaling schemes for underlay cognitive radio networks," in Proc. IEEE Int. Conf. Commun. (ICC). IEEE, Jun. 2015, pp. 1398-1403.

[31] O. Amin, W. Abediseid, and M.-S. Alouini, "Underlay cognitive radio systems with improper Gaussian signaling: Outage performance analysis," IEEE Trans. Wireless Commun., vol. 15, no. 7, Jul. 2016.

[32] M. Gaafar, O. Amin, W. Abediseid, and M.-S. Alouini, "Underlay spectrum sharing techniques with in-band full-duplex systems using improper Gaussian signaling," IEEE Trans. Wireless Commun., vol. 16 , no. 1, pp. 235-249, Jan. 2017.

[35] G. Oliveira, E. Fernandez, S. Mafra, and S. Montejo-Sánchez, "Physical layer security in cognitive radio networks using improper gaussian signaling," IEEE Commun. Lett., vol. 22, no. 9, pp. 1886-1889, Sept. 2018.
[33] O. Amin, W. Abediseid, and M.-S. Alouini, "Overlay spectrum sharing using improper Gaussian signaling," IEEE J. Sel. Areas Commun., vol. 35, no. 1, pp. 50-62, Jan. 2017.

[34] W. Hedhly, O. Amin, and M.-S. Alouini, "Interweave cognitive radio with improper gaussian signaling," in Proc. IEEE Global Commun. Conf. (GLOBECOM). IEEE, Dec. 2017, pp. 1-6.

[36] A. Patel, Z. Khan, S. Merchant, U. Desai, and L. Hanzo, "The achievable rate of interweave cognitive radio in the face of sensing errors," IEEE Access, Sept. 2016.

[37] S. Lee, T. Q. Duong, D. Benevides daCosta, D.-B. Ha, and S. Q. Nguyen, "Underlay cognitive radio networks with cooperative non-orthogonal multiple access," IET Communications, vol. 12, no. 3, pp. 359-366, 2017.

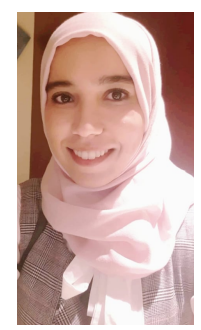

Wafa Hedhly was born in Bizerte, Tunisia. She received Telecommunication Engineering degree from the Higher School of Communications of Tunis (Sup'Com), Ariana, Tunisia, in 2017. She is currently pursuing the M.S./Ph.D.degrees with the Electrical Engineering Program,King Abdullah University of Science and Technology, Thuwal, Makkah Prov., Saudi Arabia, since 2017. Her research interests include cognitive radio, channel modeling, Terahertz communication.

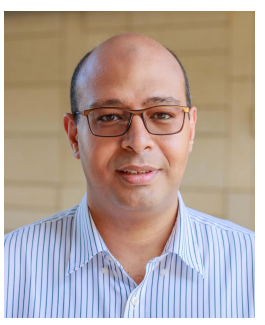

Osama Amin (S'07, M'11, SM'15) received B.Sc degree in Electrical and Electronic Engineering from Aswan University, Aswan, Egypt, in 2000, M.Sc. degree in Electrical and Electronic Engineering Engineering from Assiut University, Assiut, Egypt in 2004 and Ph.D. degree in Electrical and Computer Engineering, University of Waterloo, Waterloo, ON, Canada in 2010. In June 2012, he joined Assiut University as an Assistant Professor in the Electrical and Electronics Engineering department. He is currently a Research Scientist with the King Abdullah University of Science and Technology, Thuwal, Makkah Prov., Saudi Arabia. Dr. Amin is an associate editor for the IEEE COMMUNICATIONS LETTERS and IEEE OPEN JOURNAL OF THE COMMUNICATIONS SOCIETY. His general research interests lie in communication systems and signal processing for communications with special emphasis on wireless applications. Specific research areas include optical wireless communication, underwater optical wireless communication, Terahertz communication, Green communication, cognitive radio, and molecular communication.

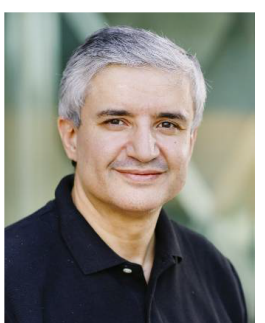

Mohamed-Slim Alouini (S'94-M'98-SM'03-F'09) was born in Tunis, Tunisia. He received the Ph.D. degree in Electrical Engineering from the California Institute of Technology (Caltech), Pasadena, CA, USA, in 1998. He served as a faculty member in the University of Minnesota, Minneapolis, MN, USA, then in the Texas A\&M University at Qatar, Education City, Doha, Qatar before joining King Abdullah University of Science and Technology (KAUST), Thuwal, Makkah Province, Saudi Arabia as a Professor of Electrical Engineering in 2009. His current research interests include the modeling, design, and performance analysis of wireless communication systems. 